\title{
Mass function and bias of dark matter halos for non-Gaussian initial conditions
}

\begin{abstract}
P. Valageas
Institut de Physique Théorique, CEA Saclay, 91191 Gif-sur-Yvette, France

e-mail: patrick.valageas@cea.fr

Received 4 June 2009 / Accepted 31 January 2010

ABSTRACT

Aims. We revisit the derivation of the mass function and the bias of dark matter halos for non-Gaussian initial conditions.

Methods. We use a steepest-descent approach to point out that exact results can be obtained for the high-mass tail of the halo mass function and the two-point correlation of massive halos. Focusing on primordial non-Gaussianity of the local type, we check that these results agree with numerical simulations.

Results. The high-mass cutoff of the halo mass function takes the same form as the one obtained from the Press-Schechter formalism, but with a linear threshold $\delta_{\mathrm{L}}$ that depends on the definition of the halo (i.e. $\delta_{\mathrm{L}} \simeq 1.59$ for a nonlinear density contrast of 200 ). We show that a simple formula, which obeys this high-mass asymptotic and uses the fit obtained for Gaussian initial conditions, matches numerical simulations while keeping the mass function normalized to unity. Next, by deriving the real-space halo two-point correlation in the spirit of Kaiser (1984, ApJ, 284, L9) and taking a Fourier transform, we obtain good agreement with simulations for the correction to the halo bias, $\Delta b_{M}\left(k, f_{\mathrm{NL}}\right)$, due to primordial non-Gaussianity. Therefore, neither the halo mass function nor the bias require an ad-hoc parameter $q$ (such as $\delta_{\mathrm{c}} \rightarrow \delta_{\mathrm{c}} \sqrt{q}$ ), provided one uses the correct linear threshold $\delta_{\mathrm{L}}$ and pays attention to halo displacements. The nonlinear real-space expression can be useful for checking that the "linearized" bias is a valid approximation. Moreover, it clearly shows how the baryon acoustic oscillation at $\sim 100 \mathrm{~h}^{-1} \mathrm{Mpc}$ is amplified by the bias of massive halos and modified by primordial non-Gaussianity. On smaller scales, $30<x<90 h^{-1} \mathrm{Mpc}$, the correction to the real-space bias roughly scales as $f_{\mathrm{NL}} b_{M}\left(f_{\mathrm{NL}}=0\right) x^{2}$. The low- $k$ behavior of the halo bias does not imply a divergent real-space correlation, so that one does not need to introduce counterterms that depend on the survey size.
\end{abstract}

Key words. gravitation - methods: analytical - large-scale structure of Universe

\section{Introduction}

Standard single-field slow-roll inflationary models predict a nearly scale-invariant and Gaussian spectrum of primordial curvature fluctuations (e.g., Bartolo et al. 2004). This agrees with current observations of the cosmic microwave background (CMB) anisotropies (Komatsu et al. 2009) and of large-scale structures (Slosar et al. 2008). Nevertheless, several inflationary models predict a potentially observable level of non-Gaussianity (e.g., Bartolo et al. 2004, for a review), so that constraining or detecting primordial non-Gaussianity is an important task for current cosmological studies. This would allow one to rule out some of the many inflationary models that have already been proposed. In particular, in many cases, the non-Gaussianity is of the local type, meaning that it only depends on the local value of Bardeen's potential $\Phi$. That is, the latter can be decomposed as

local type : $\Phi(\boldsymbol{x})=\phi(\boldsymbol{x})+f_{\mathrm{NL}}\left(\phi(\boldsymbol{x})^{2}-\left\langle\phi^{2}\right\rangle\right)$,

where $\phi$ is a Gaussian random field. Simple slow-roll inflation gives a parameter $f_{\mathrm{NL}}$ of $10^{-2}$, but this would be masked by the nonlinearities that arise from the dynamics (e.g., from the nonlinearity of Einstein's equations, see Bartolo et al. 2004) or from the physical processes involved by the observables (e.g., perturbations at recombination that affect the CMB, see Senatore et al. 2009), which give an effective $f_{\mathrm{NL}}$ close to unity. High values of $f_{\mathrm{NL}}$ can be obtained, for instance, from multifield inflation (Bartolo et al. 2002; Lyth et al. 2003), self-interactions (Falk et al. 1993), tachyonic preheating in hybrid inflation
(Barnaby \& Cline 2006), or ghost inflation (Arkani-Hamed et al. 2004). Current limits are $-9<f_{\mathrm{NL}}<111$ from CMB (Komatsu et al. 2009) and $-29<f_{\mathrm{NL}}<70$ from large-scale structures (Slosar et al. 2008).

The effects of primordial non-Gaussianity on large-scale structures can be seen, for instance, through the mass function of virialized halos, especially in the high-mass tail as the steep falloff magnifies the sensitivity to initial conditions (Lucchin \& Matarrese 1988; Colafrancesco et al. 1989; Grossi et al. 2007; Maggiore \& Riotto 2009). This allows using the X-ray luminosity function of clusters to constrain the amount of nonGaussianity (Amara \& Refregier 2004).

A second probe of non-Gaussianity is provided by the clustering of these halos, as measured through their many-body correlations. In particular, the halo two-point correlation can be significantly increased if the underlying primordial density field is non-Gaussian (Grinstein \& Wise 1986). More specifically, Dalal et al. (2008) have recently shown that primordial nonGaussianity of the local type (1) gives rises to a strongly scaledependent bias on large scales, whereas in the Gaussian case the bias is roughly constant in this range. Thus, at linear order over $f_{\mathrm{NL}}$ they obtain in Fourier space a correction of the form

$\Delta b_{M}\left(k, f_{\mathrm{NL}}\right)=f_{\mathrm{NL}}\left[b_{M}(k, 0)-1\right] \frac{3 \delta_{\mathrm{L}} \Omega_{\mathrm{m} 0} H_{0}^{2}}{c^{2} k^{2} T(k) D(z)}$,

where $b_{M}(k, 0)$ is the Gaussian-case bias (defined as the ratio of the halo and matter power spectra, $b_{M}^{2}(k)=P_{M}(k) / P(k)$, for objects of mass $M$ ). Here $\delta_{\mathrm{L}}$ is the linear matter density contrast 
associated with virialized objects (usually taken as $\delta_{\mathrm{L}} \simeq 1.686$ ), $T(k)$ the transfer function, and $D(z)$ the linear growth factor, normalized as $D(z) \rightarrow(1+z)^{-1}$ at high redshift. Then, Dalal et al. (2008) checked in numerical simulations that, in agreement with Eq. (2), the halo bias correction roughly grows as $1 / k^{2}$ at low $k$. This gives rise to a significant and specific signal that has already been used to constrain $f_{\mathrm{NL}}$ (Slosar et al. 2008).

In this article, following a previous work devoted to the Gaussian case (Valageas 2009b), we revisit the derivations of the halo mass function and of the bias for primordial nonGaussianity. Although we focus on the local type (1), our approach also applies to any non-Gaussian model where Bardeen's potential can be written as the sum of linear and quadratic terms over an auxiliary Gaussian field, that is, where $f_{\mathrm{NL}}$ becomes a convolution kernel. (It also extends to cases that contain higher order terms and multiple Gaussian fields.)

After introducing our notations and the quantities needed for our calculations in Sect. 2, we consider the halo mass function in Sect. 3. Here, our aim is to argue that the exponential cutoff of the high-mass tail can be obtained exactly from a saddle-point approach. This is equivalent to the saddle-point computation of Matarrese et al. (2000), which is often used to model the nonGaussian halo mass function. However, with a different treatment, we simultaneously derive the linear density profile of this saddle point, which allows us to check that the latter is almost insensitive to primordial non-Gaussianity, so that shell crossing is not amplified and exact results can be obtained provided one uses the correct linear density threshold, rather than the usual one. We also propose a simple recipe to match the dependence on $f_{\mathrm{NL}}$ of the high-mass tail while keeping the mass function normalized to unity. Then, in Sect. 4 we consider the two-point correlation of dark matter halos in real space, following the spirit of Kaiser (1984). Next, taking a Fourier transform we obtain the halo bias in Fourier space. Here, our aim is to show that one does not need to introduce free parameters to match the results of numerical simulations. Moreover, the nonlinear real-space expression is of interest by itself and it also allows one to check whether the "linearized" bias is valid on the range of interest. Finally, we conclude in Sect. 5.

\section{Non-Gaussian initial conditions}

We focus in this paper on non-Gaussianities of the local type, where Bardeen's potential $\Phi$ is of the form (1), with $\phi$ a Gaussian random field. On scales smaller than the Hubble radius, $\Phi$ equals minus the Newtonian gravitational potential and the Poisson equation gives in Fourier space (Slosar et al. 2008)

$\tilde{\delta}_{\mathrm{L}}(\boldsymbol{k}, z)=\alpha(k, z) \tilde{\Phi}(\boldsymbol{k}) \quad$ with $\alpha(k, z)=\frac{2 c^{2} k^{2} T(k) D(z)}{3 \Omega_{\mathrm{m} 0} H_{0}^{2}}$,

where $\delta_{\mathrm{L}}$ is the linear matter density contrast, $T(k)$ is the transfer function and $D(z)$ is the linear growth factor, normalized as $D(z) \rightarrow(1+z)^{-1}$ at high redshift. Unless stated otherwise, we normalize the Fourier transform as

$\delta_{\mathrm{L}}(\boldsymbol{x})=\int \mathrm{d} \boldsymbol{k} \mathrm{e}^{\mathrm{i} \boldsymbol{k} \cdot \boldsymbol{x}} \tilde{\delta}_{\mathrm{L}}(\boldsymbol{k})$

Note that we define $f_{\mathrm{NL}}$ by applying Eq. (1) at early times (i.e. $z=\infty$ ), which is sometimes called the "CMB convention", whereas some authors first linearly extrapolate $\Phi$ at $z=$ 0 ("LSS convention"). Thus, both conventions are related by
$f_{\mathrm{NL}}^{\mathrm{CMB}}=D(0) f_{\mathrm{NL}}^{\mathrm{LSS}}$ (Pillepich et al. 2010). Then, defining the time-dependent Gaussian field $\chi$ by

$\tilde{\chi}(\boldsymbol{k}, z)=\alpha(k, z) \tilde{\phi}(\boldsymbol{k})$

we can write the linear density field at redshift $z$ as

$$
\begin{aligned}
\tilde{\delta}_{\mathrm{L}}(\boldsymbol{k})= & \tilde{\chi}(\boldsymbol{k})+\int \mathrm{d} \boldsymbol{k}_{1} \mathrm{~d} \boldsymbol{k}_{2} \delta_{D}\left(\boldsymbol{k}_{1}+\boldsymbol{k}_{2}-\boldsymbol{k}\right) \tilde{f}_{\mathrm{NL}}^{\delta}\left(\boldsymbol{k}_{1}, \boldsymbol{k}_{2}\right) \\
& \times \tilde{\chi}\left(\boldsymbol{k}_{1}\right) \tilde{\chi}\left(\boldsymbol{k}_{2}\right),
\end{aligned}
$$

with

local type : $\tilde{f}_{\mathrm{NL}}^{\delta}\left(\boldsymbol{k}_{1}, \boldsymbol{k}_{2}\right)=f_{\mathrm{NL}} \frac{\alpha\left(\boldsymbol{k}_{1}+\boldsymbol{k}_{2}\right)}{\alpha\left(k_{1}\right) \alpha\left(k_{2}\right)}$.

This reads in real space as

$\delta_{\mathrm{L}}(\boldsymbol{x})=\chi(\boldsymbol{x})+\int \mathrm{d} \boldsymbol{x}_{1} \mathrm{~d} \boldsymbol{x}_{2} f_{\mathrm{NL}}^{\delta}\left(\boldsymbol{x} ; \boldsymbol{x}_{1}, \boldsymbol{x}_{2}\right) \chi\left(\boldsymbol{x}_{1}\right) \chi\left(\boldsymbol{x}_{2}\right)$,

where the kernel $f_{\mathrm{NL}}^{\delta}\left(\boldsymbol{x} ; \boldsymbol{x}_{1}, \boldsymbol{x}_{2}\right)$ only depends on the two vectors $\left\{x_{1}-x, x_{2}-x\right\}$

$f_{\mathrm{NL}}^{\delta}\left(x ; x_{1}, x_{2}\right)=f_{\mathrm{NL}}^{\delta}\left(x_{1}-x, x_{2}-x\right)$,

as long as the system remains statistically homogeneous, as for the local model (1). The real-space and Fourier-space kernels are related by (note the different normalization from (4))

$f_{\mathrm{NL}}^{\delta}\left(\boldsymbol{x}_{1}, \boldsymbol{x}_{2}\right)=\int \frac{\mathrm{d} \boldsymbol{k}_{1} \mathrm{~d} \boldsymbol{k}_{2}}{(2 \pi)^{6}} \mathrm{e}^{\mathrm{i} \boldsymbol{k}_{1} \cdot \boldsymbol{x}_{1}+\mathrm{i} \boldsymbol{k}_{2} \cdot \boldsymbol{x}_{2}} \tilde{f}_{\mathrm{NL}}^{\delta}\left(\boldsymbol{k}_{1}, \boldsymbol{k}_{2}\right)$.

The relationships (6) and (8) describe any homogeneous model where the Bardeen potential can be expressed as the sum of linear and quadratic terms over some Gaussian field. Thus, our analytical results also apply to other " $f_{\mathrm{NL}}$-type" models than the "local" one (1).

For $f_{\mathrm{NL}}=0$ we recover Gaussian initial conditions, $\delta_{\mathrm{L}}=\chi$, with a linear density power spectrum

$\left\langle\tilde{\chi}\left(\boldsymbol{k}_{1}\right) \tilde{\chi}\left(\boldsymbol{k}_{2}\right)\right\rangle=\delta_{D}\left(\boldsymbol{k}_{1}+\boldsymbol{k}_{2}\right) P_{\mathrm{L}}\left(k_{1}\right)$,

and a two-point linear density correlation

$$
\begin{aligned}
C_{\mathrm{L}}\left(\boldsymbol{x}_{1}, \boldsymbol{x}_{2}\right) & =\left\langle\chi\left(\boldsymbol{x}_{1}\right) \chi\left(\boldsymbol{x}_{2}\right)\right\rangle \\
& =4 \pi \int \mathrm{d} k k^{2} P_{\mathrm{L}}(k) \frac{\sin \left(k\left|\boldsymbol{x}_{2}-\boldsymbol{x}_{1}\right|\right)}{k\left|\boldsymbol{x}_{2}-\boldsymbol{x}_{1}\right|} .
\end{aligned}
$$

As usual, it is convenient to introduce the smoothed linear density contrast, $\chi_{q}(\boldsymbol{x})$, within the sphere of radius $q$ and volume $V$ around position $\boldsymbol{x}$,

$\chi_{q}(\boldsymbol{x})=\int_{V} \frac{\mathrm{d} \boldsymbol{x}^{\prime}}{V} \chi\left(\boldsymbol{x}+\boldsymbol{x}^{\prime}\right)=\int \mathrm{d} \boldsymbol{k} \mathrm{e}^{\mathrm{i} \boldsymbol{k} \cdot \boldsymbol{x}} \tilde{\chi}(\boldsymbol{k}) \tilde{W}(k q)$,

with a top-hat window that reads in Fourier space as

$\tilde{W}(k q)=\int_{V} \frac{\mathrm{d} \boldsymbol{x}}{V} \mathrm{e}^{\mathrm{i} k \cdot \boldsymbol{x}}=3 \frac{\sin (k q)-k q \cos (k q)}{(k q)^{3}}$.

Then, in the linear regime, the cross-correlation of the smoothed linear density contrasts on scales $q_{1}$ and $q_{2}$ and positions $\boldsymbol{x}_{1}$ and $\boldsymbol{x}_{2}=\boldsymbol{x}_{1}+\boldsymbol{x}$ reads as

$$
\begin{aligned}
\sigma_{q_{1}, q_{2}}^{2}(x) & =\left\langle\chi_{q_{1}}\left(\boldsymbol{x}_{1}\right) \chi_{q_{2}}\left(\boldsymbol{x}_{1}+\boldsymbol{x}\right)\right\rangle \\
& =4 \pi \int \mathrm{d} k k^{2} P_{\mathrm{L}}(k) \tilde{W}\left(k q_{1}\right) \tilde{W}\left(k q_{2}\right) \frac{\sin (k x)}{k x}
\end{aligned}
$$


In particular, $\sigma_{q}=\sigma_{q, q}(0)$ is the usual rms linear density contrast on scale $q$. Then, in the non-Gaussian case we define the initial conditions by the same power spectrum (11) for the field $\chi$ and we vary the parameter $f_{\mathrm{NL}}$ (for the local type (7)). We still define the variance $\sigma^{2}$ as in Eq. (15) from the Gaussian field $\chi$.

In the following sections, where we define dark matter halos as spherical overdensities, we shall need the average of the kernel $f_{\mathrm{NL}}^{\delta}\left(\boldsymbol{x} ; \boldsymbol{x}_{1}, \boldsymbol{x}_{2}\right)$ over spherical cells, weighted by the linear correlation (12). Thus, omitting the superscript $\delta$ for simplicity, we define the quantity

$$
\begin{aligned}
f_{q ; q_{1}, q_{2}}\left(\boldsymbol{x} ; \boldsymbol{x}_{1}, \boldsymbol{x}_{2}\right)= & \int_{V} \frac{\mathrm{d} \boldsymbol{q}}{V} \int_{V_{1}} \frac{\mathrm{d} \boldsymbol{q}_{1}}{V_{1}} \int_{V_{2}} \frac{\mathrm{d} \boldsymbol{q}_{2}}{V_{2}} \int \mathrm{d} \boldsymbol{q}_{1}^{\prime} \mathrm{d} \boldsymbol{q}_{2}^{\prime} \\
& \times f_{\mathrm{NL}}^{\delta}\left(\boldsymbol{q} ; \boldsymbol{q}_{1}^{\prime}, \boldsymbol{q}_{2}^{\prime}\right) C_{\mathrm{L}}\left(\boldsymbol{q}_{1}^{\prime}, \boldsymbol{q}_{1}\right) C_{\mathrm{L}}\left(\boldsymbol{q}_{2}^{\prime}, \boldsymbol{q}_{2}\right),
\end{aligned}
$$

where the spheres of volumes $V, V_{1}$ and $V_{2}$, and radii $q, q_{1}$ and $q_{2}$, are centered on the points $\boldsymbol{x}, \boldsymbol{x}_{1}$ and $\boldsymbol{x}_{2}$. In Eq. (16) the coordinates $\boldsymbol{q}_{1}^{\prime}$ and $\boldsymbol{q}_{2}^{\prime}$ are integrated over all space. In terms of the Fourier kernel $\tilde{f}_{\mathrm{NL}}^{\delta}$ this reads as

$$
\begin{aligned}
f_{q ; q_{1}, q_{2}}\left(\boldsymbol{x} ; \boldsymbol{x}_{1}, \boldsymbol{x}_{2}\right)= & \int \mathrm{d} \boldsymbol{k}_{1} \mathrm{~d} \boldsymbol{k}_{2} \tilde{f}_{\mathrm{NL}}^{\delta}\left(\boldsymbol{k}_{1}, \boldsymbol{k}_{2}\right) P_{\mathrm{L}}\left(k_{1}\right) P_{\mathrm{L}}\left(k_{2}\right) \\
& \times \tilde{W}\left(k_{1} q_{1}\right) \tilde{W}\left(k_{2} q_{2}\right) \tilde{W}\left(\left|\boldsymbol{k}_{1}+\boldsymbol{k}_{2}\right| q\right) \\
& \times \mathrm{e}^{\mathrm{i} \boldsymbol{k}_{1} \cdot\left(\boldsymbol{x}_{1}-\boldsymbol{x}\right)+\mathrm{i} \boldsymbol{k}_{2} \cdot\left(\boldsymbol{x}_{2}-\boldsymbol{x}\right)} .
\end{aligned}
$$

Thanks to statistical homogeneity and isotropy, the kernel $\tilde{f}_{\mathrm{NL}}^{\delta}\left(\boldsymbol{k}_{1}, \boldsymbol{k}_{2}\right)$ only depends on the lengths $k_{1}, k_{2}$, and on the angle between both vectors. Then, for spheres that are centered on the same point (i.e. $\boldsymbol{x}=\boldsymbol{x}_{1}=\boldsymbol{x}_{2}$ ), Eq. (17) simplifies as

$$
\begin{aligned}
\boldsymbol{x}=\boldsymbol{x}_{1}=\boldsymbol{x}_{2}: \quad f_{q ; q_{1}, q_{2}}= & 8 \pi^{2} \int_{0}^{\infty} \mathrm{d} k_{1} k_{1}^{2} P_{\mathrm{L}}\left(k_{1}\right) \tilde{W}\left(k_{1} q_{1}\right) \\
& \times \int_{0}^{\infty} \mathrm{d} k_{2} k_{2}^{2} P_{\mathrm{L}}\left(k_{2}\right) \tilde{W}\left(k_{2} q_{2}\right) \\
& \times \int_{-1}^{1} \mathrm{~d} \mu \tilde{W}(k q) \tilde{f}_{\mathrm{NL}}^{\delta}\left(k_{1}, k_{2}, \mu\right),
\end{aligned}
$$

which does not depend on the position $\boldsymbol{x}$ of the sphere. Here we defined $\mu=\left(\boldsymbol{k}_{1} \cdot \boldsymbol{k}_{2}\right) /\left(k_{1} k_{2}\right)$, and $k^{2}=k_{1}^{2}+k_{2}^{2}+2 k_{1} k_{2} \mu$. On the other hand, when we consider the two-point correlation of dark matter halos, the three spheres in Eq. (16) are chosen among two possible spheres $V_{a}$ and $V_{b}$, separated by a distance $x$. Then, we need the two quantities,

$$
\begin{aligned}
V=V_{a}, V_{1}= & V_{2}=V_{b}: \quad f_{a ; b b}(x)=8 \pi^{2} \int_{0}^{\infty} \mathrm{d} k_{1} k_{1}^{2} P_{\mathrm{L}}\left(k_{1}\right) \\
& \times \tilde{W}\left(k_{1} q_{b}\right) \int_{0}^{\infty} \mathrm{d} k_{2} k_{2}^{2} P_{\mathrm{L}}\left(k_{2}\right) \tilde{W}\left(k_{2} q_{b}\right) \\
& \times \int_{-1}^{1} \mathrm{~d} \mu \tilde{W}\left(k q_{a}\right) \tilde{f}_{\mathrm{NL}}^{\delta}\left(k_{1}, k_{2}, \mu\right) \frac{\sin (k x)}{k x},
\end{aligned}
$$

and

$$
\begin{aligned}
V_{1}=V_{a}, V= & V_{2}=V_{b}: \quad f_{b ; a b}(x)=8 \pi^{2} \int_{0}^{\infty} \mathrm{d} k_{1} k_{1}^{2} P_{\mathrm{L}}\left(k_{1}\right) \\
& \times \tilde{W}\left(k_{1} q_{a}\right) \frac{\sin \left(k_{1} x\right)}{k_{1} x} \int_{0}^{\infty} \mathrm{d} k_{2} k_{2}^{2} P_{\mathrm{L}}\left(k_{2}\right) \tilde{W}\left(k_{2} q_{b}\right) \\
& \times \int_{-1}^{1} \mathrm{~d} \mu \tilde{W}\left(k q_{b}\right) \tilde{f}_{\mathrm{NL}}^{\delta}\left(k_{1}, k_{2}, \mu\right)
\end{aligned}
$$

Their Fourier transforms with respect to the separation $\boldsymbol{x}$ read as

$$
\begin{aligned}
\tilde{f}_{a ; b b}(k)= & \int \mathrm{d} \boldsymbol{k}_{1} \mathrm{~d} \boldsymbol{k}_{2} \delta_{D}\left(\boldsymbol{k}_{1}+\boldsymbol{k}_{2}-\boldsymbol{k}\right) P_{\mathrm{L}}\left(k_{1}\right) P_{\mathrm{L}}\left(k_{2}\right) \\
& \times \tilde{W}\left(k_{1} q_{b}\right) \tilde{W}\left(k_{2} q_{b}\right) \tilde{W}\left(k q_{a}\right) \tilde{f}_{\mathrm{NL}}^{\delta}\left(\boldsymbol{k}_{1}, \boldsymbol{k}_{2}\right),
\end{aligned}
$$

and

$$
\begin{aligned}
\tilde{f}_{b ; a b}(k)= & P_{\mathrm{L}}(k) \tilde{W}\left(k q_{a}\right) \int \mathrm{d} \boldsymbol{k}_{1} P_{\mathrm{L}}\left(k_{1}\right) \tilde{W}\left(k_{1} q_{b}\right) \\
& \times \tilde{W}\left(\left|\boldsymbol{k}_{1}+\boldsymbol{k}\right| q_{b}\right) \tilde{f}_{\mathrm{NL}}^{\delta}\left(\boldsymbol{k}, \boldsymbol{k}_{1}\right) .
\end{aligned}
$$

\section{Mass function of dark matter halos}

We now extend the analysis of Valageas (2009b) to obtain the mass function of dark matter halos for non-Gaussian initial conditions.

\subsection{Rare-event saddle point for the density distribution}

In a fashion similar to Valageas (2009b), we note that the exponential falloffs of the high-mass tail of the halo mass function $n(M)$, and of the overdensity tail of the linear density contrast distribution $\mathcal{P}_{\mathrm{L}}\left(\delta_{\mathrm{L} q}\right)$, can be exactly obtained from the constrained maximum,

rare events : $\mathcal{P}_{\mathrm{L}}\left(\delta_{\mathrm{L}}\right) \sim \max _{\left\{\chi[q] \mid \delta_{\mathrm{Lq}}=\delta_{\mathrm{L}}\right\}} \mathrm{e}^{-\frac{1}{2} \chi \cdot C_{\mathrm{L}}^{-1} \cdot \chi}$.

Here we introduced the probability distribution $\mathcal{P}_{\mathrm{L}}\left(\delta_{\mathrm{L} q}\right)$ of the smoothed linear density contrast within the sphere of radius $q$, which we can take centered on the origin,

$\delta_{\mathrm{L} q}=\int_{V} \frac{\mathrm{d} \boldsymbol{q}^{\prime}}{V} \delta_{\mathrm{L}}\left(\boldsymbol{q}^{\prime}\right)=\int \mathrm{d} \boldsymbol{k} \tilde{\delta}_{\mathrm{L}}(\boldsymbol{k}) \tilde{W}(k q)$.

In the Gaussian case, $f_{\mathrm{NL}}=0$, we simply have $\delta_{\mathrm{L} q}=\chi_{q}$, as defined in Eq. (13), and the probability distribution $\mathcal{P}_{\mathrm{L}}$ is a Gaussian. Then, as stressed in Valageas (2009a,b), in the limit of rare events (e.g., at fixed density contrast $\delta_{\mathrm{L} q}$ in the largescale or high-mass limit $q \rightarrow \infty$ ), the tails of the distribution $\mathcal{P}_{\mathrm{L}}$ are obtained from Eq. (23), where we maximize the statistical weight $\mathrm{e}^{-\left(\chi \cdot C_{\mathrm{L}}^{-1} \cdot \chi\right) / 2}$ of the Gaussian field $\chi$, under the constraint that the smoothed linear density contrast $\delta_{\mathrm{L} q}$ is equal to the value of interest. Equation (23) only gives the leading-order exponential falloff. Subleading terms, such as power-law prefactors, may be obtained by expanding around the saddle-point $\chi$, within a steepest-descent method. This also provides the probability distribution $\mathcal{P}(\delta)$ of the nonlinear density contrast $\delta$ on scale $r$ in the quasi-linear regime, as well as the cumulant generating function (Valageas 2002a,b, 2009a,b) ${ }^{1}$. As pointed out in Valageas (2009b), this also gives the high-mass tail of the halo mass function, if halos are defined as spherical overdensities with a fixed nonlinear density threshold $\delta_{r}$. Then, the halo mass $M$ and radius $r$ are related to the Lagrangian radius $q$ and linear density threshold $\delta_{\mathrm{L} q}$ through

$q^{3}=\left(1+\delta_{r}\right) r^{3}$, with $\delta_{r}=\mathcal{F}\left(\delta_{\mathrm{L} q}\right)$,

and

$M=\bar{\rho}_{\mathrm{m}} \frac{4 \pi}{3} q^{3}$,

where the function $\mathcal{F}$ describes the spherical collapse dynamics. This holds as long as shell-crossing has not extended beyond

\footnotetext{
1 As shown in Valageas (2009a), for the closely related adhesion model, where the same procedure can be applied, one can explicitly check that the asymptotic results obtained by this approach agree with the complete distribution $\mathcal{P}(\delta)$ that is exactly known for two cases (Brownian and white-noise linear velocity in $1 \mathrm{D}$, corresponding to a power-law linear density power spectrum with $n=-2$ and $n=0$ ).
} 
radius $r$, so that the usual spherical collapse dynamics at constant mass is valid. This yields an upper bound $\delta_{+}$for the nonlinear density threshold that can be used to define halos to take advantage of the exact asymptotic tail (23). For the preferred $\Lambda \mathrm{CDM}$ model this gives $\delta_{+} \sim 200$ for $M \sim 10^{15} h^{-1} M_{\odot}$ up to $\delta_{+} \sim 600$ for $M \sim 10^{11} h^{-1} M_{\odot}$ (the dependence on mass is due to the change of slope of the matter power spectrum $P_{\mathrm{L}}(k)$ ).

In the Gaussian case, the constrained weight (23) simply reads as $\mathrm{e}^{-\delta_{\mathrm{L} q}^{2} /\left(2 \sigma_{q}^{2}\right)}$, and we recover the exponential tail of the usual Press-Schechter mass function (Press \& Schechter 1974), except that the standard threshold $\delta_{\mathrm{c}} \simeq 1.686$ must be replaced by $\delta_{\mathrm{L}}=\mathcal{F}^{-1}(\delta)$, with $\delta_{\mathrm{L}} \simeq 1.59$ for $\delta=200$. In the non-Gaussian case, that is for $f_{\mathrm{NL}} \neq 0$, we can compute the weight (23) by using a Lagrange multiplier $\lambda$. Thus, we define the action $\mathcal{S}[\chi, \lambda]$,

$\mathcal{S}[\chi, \lambda]=\lambda\left(\delta_{\mathrm{L}}-\delta_{\mathrm{L} q}[\chi]\right)+\frac{1}{2} \chi \cdot C_{\mathrm{L}}^{-1} \cdot \chi$

where $\delta_{\mathrm{L} q}[\chi]$ is the nonlinear functional that affects to the initial condition defined by the Gaussian field $\chi$ the linear density contrast $\delta_{\mathrm{L} q}$ within the sphere of radius $q$, obtained through Eq. (8). Then, we must look for the saddle point of the action (27), with respect to both $\chi$ and $\lambda$. Thus, differentiating the action (27) with respect to $\chi(\boldsymbol{q})$ and multiplying by the operator $C_{\mathrm{L}}$ gives

$\chi(\boldsymbol{q})=\lambda C_{\mathrm{L}}\left(\boldsymbol{q}, \boldsymbol{q}^{\prime}\right) \cdot \frac{\mathcal{D} \delta_{\mathrm{L} q}}{\mathcal{D} \chi\left(\boldsymbol{q}^{\prime}\right)}$,

whence, using Eq. (8),

$$
\begin{aligned}
\chi(\boldsymbol{q})= & \lambda \int_{V} \frac{\mathrm{d} \boldsymbol{q}^{\prime}}{V} C_{\mathrm{L}}\left(\boldsymbol{q}, \boldsymbol{q}^{\prime}\right)+2 \lambda \int_{V} \frac{\mathrm{d} \boldsymbol{q}^{\prime}}{V} \int \mathrm{d} \boldsymbol{q}_{1} \mathrm{~d} \boldsymbol{q}_{2} \\
& \times f_{\mathrm{NL}}^{\delta}\left(\boldsymbol{q}^{\prime} ; \boldsymbol{q}_{1}, \boldsymbol{q}_{2}\right) C_{\mathrm{L}}\left(\boldsymbol{q}, \boldsymbol{q}_{2}\right) \chi\left(\boldsymbol{q}_{1}\right) .
\end{aligned}
$$

Differentiating the action (27) with respect to $\lambda$ gives the constraint

$\delta_{\mathrm{L}}=\delta_{\mathrm{L} q}[\chi]$,

whence

$\delta_{\mathrm{L}}=\int_{V} \frac{\mathrm{d} \boldsymbol{q}}{V}\left[\chi(\boldsymbol{q})+\int \mathrm{d} \boldsymbol{q}_{1} \mathrm{~d} \boldsymbol{q}_{2} f_{\mathrm{NL}}^{\delta}\left(\boldsymbol{q} ; \boldsymbol{q}_{1}, \boldsymbol{q}_{2}\right) \chi\left(\boldsymbol{q}_{1}\right) \chi\left(\boldsymbol{q}_{2}\right)\right]$.

Next, we solve the system (29)-(31) as a perturbative series over the non-Gaussianity kernel $f_{\mathrm{NL}}^{\delta}$ (i.e. over powers of the parameter $\left.f_{\mathrm{NL}}\right)$. At order zero we recover the Gaussian saddle-point,

$\chi^{(0)}(\boldsymbol{q})=\lambda^{(0)} \int_{V} \frac{\mathrm{d} \boldsymbol{q}^{\prime}}{V} C_{\mathrm{L}}\left(\boldsymbol{q}, \boldsymbol{q}^{\prime}\right)$,

$\lambda^{(0)}=\frac{\delta_{\mathrm{L}}}{\sigma_{q}^{2}}$.

At first order we obtain

$$
\begin{aligned}
\chi^{(1)}(\boldsymbol{q})= & \lambda^{(1)} \int_{V} \frac{\mathrm{d} \boldsymbol{q}^{\prime}}{V} C_{\mathrm{L}}\left(\boldsymbol{q}, \boldsymbol{q}^{\prime}\right)+2 \lambda^{(0) 2} \int_{V} \frac{\mathrm{d} \boldsymbol{q}^{\prime} \mathrm{d} \boldsymbol{q}_{1}^{\prime}}{V^{2}} \\
& \times \int \mathrm{d} \boldsymbol{q}_{1} \mathrm{~d} \boldsymbol{q}_{2} f_{\mathrm{NL}}^{\delta}\left(\boldsymbol{q}^{\prime} ; \boldsymbol{q}_{1}, \boldsymbol{q}_{2}\right) C_{\mathrm{L}}\left(\boldsymbol{q}, \boldsymbol{q}_{2}\right) C_{\mathrm{L}}\left(\boldsymbol{q}_{1}, \boldsymbol{q}_{1}^{\prime}\right),
\end{aligned}
$$

$\lambda^{(1)}=-3 \frac{\delta_{\mathrm{L}}^{2}}{\sigma_{q}^{6}} f_{q ; q q}$,

where the quantity $f_{q ; q q}$ is given by Eq. (18).

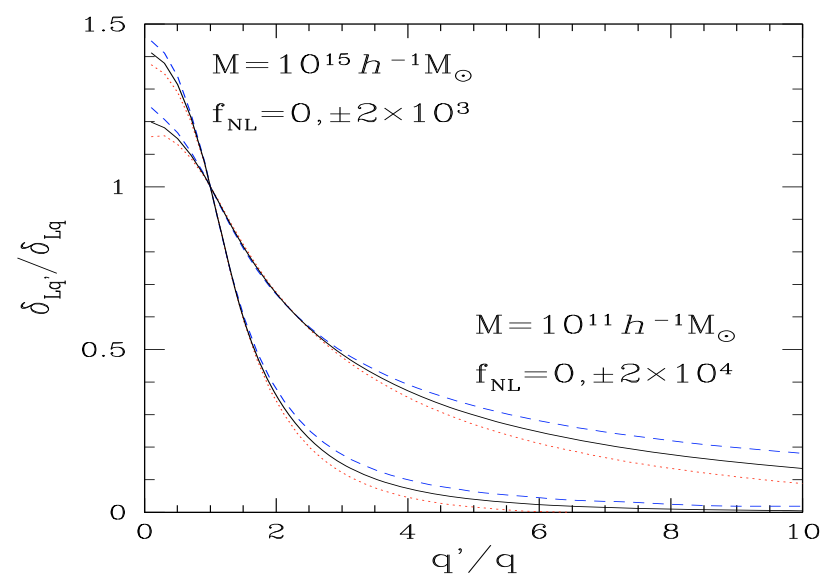

Fig. 1. The radial profile $(36,37)$ of the linear density contrast $\delta_{\mathrm{L} q^{\prime}}$ of the saddle point of the action $\mathcal{S}[\chi, \lambda]$. We show the profiles obtained with a $\Lambda$ CDM cosmology for the masses $M=10^{11}$ and $10^{15} h^{-1} M_{\odot}$. A larger mass corresponds to a lower ratio $\delta_{\mathrm{L} q^{\prime}} / \delta_{\mathrm{L} q}$ at large radii $q^{\prime} / q>1$. We show our results for the Gaussian case (solid line), positive $f_{\mathrm{NL}}$ (dashed line) and negative $f_{\mathrm{NL}}$ (dotted line), for the local model (7).

From Eqs. (32)-(35) we also obtain the radial linear density profile of the saddle-point, up to first order,

$\delta_{\mathrm{L} q^{\prime}}^{(0)}=\frac{\delta_{\mathrm{L}}}{\sigma_{q}^{2}} \sigma_{q, q^{\prime}}^{2}$,

$\delta_{\mathrm{L} q^{\prime}}^{(1)}=\frac{\delta_{\mathrm{L}}^{2}}{\sigma_{q}^{4}}\left[f_{q^{\prime} ; q q}+2 f_{q ; q q^{\prime}}-3 \frac{\sigma_{q, q^{\prime}}^{2}}{\sigma_{q}^{2}} f_{q ; q q}\right]$.

We can check that at $q^{\prime}=q$ it verifies the constraint (30), and at order zero we recover the Gaussian profile (Valageas 2009b). Equations $(36,37)$ give the integrated density profile, that is, $\delta_{\mathrm{L} q^{\prime}}$ is the mean linear density contrast within the Lagrangian radius $q^{\prime}$. The local linear density contrast at radius $q^{\prime}, \delta_{\mathrm{L}}\left(q^{\prime}\right)$, is given by

$3 q^{\prime 3} \delta_{\mathrm{L}}\left(q^{\prime}\right)=\frac{\partial}{\partial q^{\prime}}\left(q^{\prime 3} \delta_{\mathrm{L} q^{\prime}}\right)$

whence

$\delta_{\mathrm{L}}^{(0)}\left(q^{\prime}\right)=\frac{\delta_{\mathrm{L}}}{\sigma_{q}^{2}} \sigma_{q, 0}^{2}\left(q^{\prime}\right)$

$\delta_{\mathrm{L}}^{(1)}\left(q^{\prime}\right)=\frac{\delta_{\mathrm{L}}^{2}}{\sigma_{q}^{4}}\left[f_{0 ; q q}\left(q^{\prime}\right)+2 f_{q ; 0 q}\left(q^{\prime}\right)-3 \frac{\sigma_{q, 0}^{2}\left(q^{\prime}\right)}{\sigma_{q}^{2}} f_{q ; q q}\right]$

where $f_{0 ; q q}\left(q^{\prime}\right)$ and $f_{q ; 0 q}\left(q^{\prime}\right)$ are given by Eqs. $(19,20)$.

We show in Fig. 1 the integrated linear density profile (36, 37) obtained for the masses $M=10^{11}$ and $10^{15} h^{-1} M_{\odot}$, for a $\Lambda \mathrm{CDM}$ cosmology. The dependence on mass is due to the change of slope of the linear power spectrum with scale. We plot our results for the Gaussian case $\left(f_{\mathrm{NL}}=0\right.$, solid lines), large positive $f_{\mathrm{NL}}\left(f_{\mathrm{NL}}=2 \times 10^{3}\right.$ and $f_{\mathrm{NL}}=2 \times 10^{4}$, dashed lines $)$ and large negative $f_{\mathrm{NL}}\left(f_{\mathrm{NL}}=-2 \times 10^{3}\right.$ and $f_{\mathrm{NL}}=-2 \times$ $10^{4}$, dotted lines), for the local model (7). Thus, we can see that a positive $f_{\mathrm{NL}}$ increases the relative density (i.e. with respect to the density at radius $q$ ) both at small and large radii. The very large values of $f_{\mathrm{NL}}$ required to be able to distinguish the curves in the figure imply that for realistic cases $\left(\left|f_{\mathrm{NL}}\right|<100\right)$ the perturbation of the density profile is very small. Therefore, the values of the upper boundary $\delta_{+}$, which marks the onset of shell-crossing, obtained in Valageas (2009b) for the Gaussian 
case remain valid up to a very good accuracy. We can note that to obtain a similar deviation from the Gaussian profile we need a larger value of the parameter $f_{\mathrm{NL}}$ on a smaller scale. This can be understood from the expression (7), which scales as $\tilde{f}^{\delta} \sim f_{\mathrm{NL}} / \alpha(k) \propto f_{\mathrm{NL}} /\left(k^{2} T(k)\right)$ and grows as $k^{-2}$ on very large scales. The same behavior (i.e. a higher sensitivity to local-type non-Gaussianity on large scales) is obtained for the bias of dark matter halos, see Eq. (2) above and Sect. 4 below.

Next, we define the constrained weight (23) as

$\Gamma=\frac{1}{2} \chi \cdot C_{\mathrm{L}}^{-1} \cdot \chi$

at the relevant saddle-point $\chi$. This gives, up to first order,

$\Gamma^{(0)}=\frac{1}{2} \chi^{(0)} \cdot C_{\mathrm{L}}^{-1} \cdot \chi^{(0)}=\frac{\delta_{\mathrm{L}}^{2}}{2 \sigma_{q}^{2}}$,

$\Gamma^{(1)}=\chi^{(0)} \cdot C_{\mathrm{L}}^{-1} \cdot \chi^{(1)}=-\frac{\delta_{\mathrm{L}}^{3}}{\sigma_{q}^{6}} f_{q ; q q}$.

Therefore, the tails of the probability distribution (23) read as

$\mathcal{P}_{\mathrm{L}}\left(\delta_{\mathrm{L}}\right) \sim \mathrm{e}^{-\frac{\delta_{\mathrm{L}}^{2}}{2 \sigma_{q}^{2}}+\frac{\delta_{\mathrm{L}}^{3}}{\sigma_{q}^{6}} f_{q ; q q}}=\mathrm{e}^{-\frac{\delta_{\mathrm{L}}^{2}}{2 \sigma_{q}^{2}}\left(1-\frac{\delta_{\mathrm{L}}}{3} S_{3}^{(1)}\right)}$,

where we introduced the skewness of the linear density contrast, at first order over $f_{\mathrm{NL}}$,

$S_{3}^{(1)}=\left[\frac{\left\langle\delta_{\mathrm{L} q}^{3}\right\rangle}{\left\langle\delta_{\mathrm{L} q}^{2}\right\rangle^{2}}\right]^{(1)}=6 \frac{f_{q ; q q}}{\sigma_{q}^{4}}$.

Indeed, from Eq. (8) we have at first order

$$
\begin{aligned}
\left\langle\delta_{\mathrm{L} q}^{3}\right\rangle^{(1)}= & 3 \int_{V} \frac{\mathrm{d} \boldsymbol{q}_{1} \mathrm{~d} \boldsymbol{q}_{2} \mathrm{~d} \boldsymbol{q}_{3}}{V^{3}} \int \mathrm{d} \boldsymbol{q}_{1}^{\prime} \mathrm{d} \boldsymbol{q}_{2}^{\prime} f_{\mathrm{NL}}^{\delta}\left(\boldsymbol{q}_{3} ; \boldsymbol{q}_{1}^{\prime} \boldsymbol{q}_{2}^{\prime}\right) \\
& \times\left\langle\chi\left(\boldsymbol{q}_{1}\right) \chi\left(\boldsymbol{q}_{2}\right) \chi\left(\boldsymbol{q}_{1}^{\prime}\right) \chi\left(\boldsymbol{q}_{2}^{\prime}\right)\right\rangle \\
= & 6 f_{q ; q q} .
\end{aligned}
$$

Hereafter, since $S_{3}^{(0)}=0$, we simply note $S_{3}=S_{3}^{(1)}$.

\subsection{Mass function}

Following the Press-Schechter approach (Press \& Schechter 1974), the mass function that is obtained in the Gaussian case from the probability distribution $\mathcal{P}_{\mathrm{L}}\left(\delta_{\mathrm{L} q}\right)$ reads as

$n\left(M, f_{\mathrm{NL}}=0\right) \mathrm{d} M=\frac{\bar{\rho}_{\mathrm{m}}}{M} f(v) \frac{\mathrm{d} v}{v}$,

with (using the subscript "PS" to distinguish the Press-Schechter prediction)

$f_{\mathrm{PS}}(v)=\sqrt{\frac{2}{\pi}} v \mathrm{e}^{-v^{2} / 2}$

and

$v=\frac{\delta_{\mathrm{L}}}{\sigma(M)}$.

Here $\sigma(M)=\sigma_{q}$, where the Lagrangian scale $q$ is related to $M$ by Eq. (26). As stressed in Valageas (2009b), the linear threshold $\delta_{\mathrm{L}}$ in Eq. (50) must be defined as $\mathcal{F}^{-1}(\delta)$, as in Eq. (25), which gives $\delta_{\mathrm{L}} \simeq 1.59$ for $\delta=200$. Then, the exponential tail of (49) is exact,

$v \rightarrow \infty: \ln [f(v)] \sim-\frac{v^{2}}{2}$, but the power-law prefactor and the low-mass tail of (49) have no reason to be valid (and numerical simulations indeed show that they are not exact). Then, in order to match numerical simulations, one needs to use fitting formulae. One such fit to simulations, which obeys the exact tail (51), is (Valageas 2009b)

$f(v)=0.5\left[(0.6 v)^{2.5}+(0.62 v)^{0.5}\right] \mathrm{e}^{-v^{2} / 2}$.

Both mass functions (49) and (52) satisfy the normalization

$\int_{0}^{\infty} \frac{\mathrm{d} v}{v} f(v)=1$

which ensures that all the mass is contained in such halos:

$\int_{0}^{\infty} M n(M) \mathrm{d} M=\bar{\rho}_{\mathrm{m}}$.

In the non-Gaussian case, that is $f_{\mathrm{NL}} \neq 0$, we may estimate the halo mass function by multiplying the Gaussian one by the corrective factor obtained in Eq. (44),

$f_{\mathrm{NL}} \neq 0: \quad n\left(M, f_{\mathrm{NL}}\right)=n(M, 0) \mathrm{e}^{S_{3} \delta_{\mathrm{L}}^{3} /\left(6 \sigma_{q}^{2}\right)}$.

As explained above, this yields the exact high-mass tail (up to first order over $f_{\mathrm{NL}}$ ) but it is not expected to hold for the lowmass tail. In particular, this gives a non-Gaussian mass function that does not obey the normalization (54). In order to satisfy Eq. (54), while keeping the high-mass tail of Eq. (55), a simple procedure is to make use of the scaling (48) and of the normalization (53). Thus, modifying the relationship (50) as (see Eq. (44))

$\mu=\frac{\delta_{\mathrm{L}}}{\sigma(M)} \sqrt{1-\frac{\delta_{\mathrm{L}}}{3} S_{3}(M)}$,

we may use for the non-Gaussian mass function

$f_{\mathrm{NL}} \neq 0: \quad n\left(M, f_{\mathrm{NL}}\right) \mathrm{d} M=\frac{\bar{\rho}_{\mathrm{m}}}{M} f(\mu) \frac{\mathrm{d} \mu}{\mu}$,

where we use the same scaling function $f$ as for the Gaussian case (48). This recovers the high-mass tail (55) and satisfies the normalization (54). Note that Afshordi \& Tolley (2008) have recently proposed a similar rescaling as (56), which they reinterprate as a modified effective variance $\sigma_{q}^{2}$. However, to obtain such a rescaling they use some approximations, such as the decoupling between the local values of the fields $\phi(\boldsymbol{x})$ and $\chi(\boldsymbol{x})$, in the spirit of a peak-background split approximation, whereas the derivation presented here is asymptotically exact. Equation (57) yields for the ratio of both mass functions at fixed mass $M$,

$\frac{n\left(M, f_{\mathrm{NL}}\right)}{n(M, 0)}=\frac{f(\mu) / \mu}{f(v) / v} \frac{\mathrm{d} \mu}{\mathrm{d} v}$.

Using Eqs. (50), (56), we obtain

$\frac{\mathrm{d} \mu}{\mathrm{d} v}=\sqrt{1-\frac{\delta_{\mathrm{L}}}{3} S_{3}}+\frac{\sigma \delta_{\mathrm{L}}}{6 \sqrt{1-\frac{\delta_{\mathrm{L}}}{3} S_{3}}} \frac{\mathrm{d} S_{3}}{\mathrm{~d} \sigma}$

If we use the Press-Schechter mass function (49), Eqs. (57)-(59) give back the result obtained in Matarrese et al. (2000), except for the fact that we use $\delta_{\mathrm{L}} \simeq 1.59$ instead of 1.686 as explained above.

Note that Matarrese et al. (2000) also use a saddle-point approach to derive the halo mass function for non-Gaussian initial conditions, expanding at linear order over $f_{\mathrm{NL}}$ (or $S_{3}$ ), 
so that their computation is equivalent to the one described above. However, since they first compute the cumulant generating function $(W(\lambda)$ in their notations, or $\varphi(y)$ in Valageas 2009 b), the constraint (30) is expressed through a Dirac function, written as an exponential by introducing an auxiliary variable $\lambda$, so that they can first integrate over the Gaussian field $\chi$ and next expand over $f_{\mathrm{NL}}$ the expression obtained for $W(\lambda)$. The somewhat simpler method described in this article has the advantage of simultaneously giving the density profile (36), (37) of the underlying saddle point. As explained above in Fig. 1, this allows us to check that realistic amounts of primordial nonGaussianity have a negligible effect on this profile, so that the onset of shell-crossing appears for almost the same nonlinear density threshold $\delta_{+}$. This ensures that the rare-event and highmass tails (44) and (55) are exact (at leading order), as long as halos are defined by a nonlinear threshold $\delta \sim 200$ below the upper bound $\delta_{+}$. The simplicity of the method presented in this article also allows a straightforward application to two-point distributions, as shown in Sect. 4 below, or to more complex primordial non-Gaussianities, which may involve several fields or higher order polynomials as in Eq. (95) below.

Another approach presented in Lo Verde et al. (2008) is to use the Edgeworth expansion, which writes the probability distribution $\mathcal{P}_{\mathrm{L}}\left(\delta_{\mathrm{L}}\right)$ as a series over the cumulants of the nonGaussian variable $\delta_{\mathrm{L}}$. In practice, one truncates at the lowest order beyond the Gaussian, that is, at the third cumulant $\left\langle\delta_{\mathrm{L}}^{3}\right\rangle_{\mathrm{c}}$ described by $S_{3}$. Thus, expanding the exponentials (44) and (55) one recovers the results of Lo Verde et al. (2008) at large $v$ (i.e. low $\sigma_{q}$ ). However, in the rare-event limit, where computations rest on firm grounds as explained above, the Edgeworth expansion does not fare very well. In particular, although we only derived the saddle-point $\chi$ and the argument $\Gamma$ of the exponential up to linear order over $f_{\mathrm{NL}}$, see Eqs. (42), (43), it is best to keep the exponential as in Eqs. (55) or (57). Indeed, in the rare-event and small- $f_{\mathrm{NL}}$ limits, the tail (55) can be a good approximation even when the corrective factor $S_{3} \delta_{\mathrm{L}}^{3} /\left(6 \sigma_{q}^{2}\right)$ is much larger than unity (i.e. we can have the hierarchy $\Gamma^{(0)} \gg \Gamma^{(1)} \gg 1 \gg \Gamma^{(2)}$ ).

Since the low-mass tail (49) does not match numerical simulations, it is sometimes proposed to keep the ratio (58) given by the Press-Schechter mass function, and to multiply the fit from simulations of the Gaussian mass function by this factor (Grossi et al. 2007, 2009; Lo Verde et al. 2008). However, this procedure clearly violates the normalization condition (54). Therefore, we suggest to use (58) with the fitting formula obtained from Gaussian simulations, that is, to use Eq. (57), which automatically satisfies the normalization (54). However, there is no reason to expect that the low-mass tail can be exactly recovered by any such procedure, even though by construction it gives the right behavior for the Gaussian case, as the low-mass slope might also depend on $f_{\mathrm{NL}}$ in some specific manner.

We compare in Fig. 2 our results for the halo mass function with numerical simulations from Grossi et al. (2009). They use the LSS convention for $f_{\mathrm{NL}}$, so that in terms of the CMB convention used in this article this corresponds to $f_{\mathrm{NL}} \simeq \pm 151$. We show the ratio of the non-Gaussian mass function to the Gaussian one, as given by the multiplicative factor (55), the ratio (58) computed with the Press-Schechter mass function (44) or with the fitting function (52). In agreement with Grossi et al. (2009), we find that the ratio (58) computed with the Press-Schechter mass function, which also corresponds to the result of Matarrese et al. (2000) (but with $\delta_{\mathrm{L}} \simeq 1.59$ instead of 1.686 as explained above), agrees reasonably well with simulations. Using the fitting function (52) or the simple multiplicative factor (55) yields close
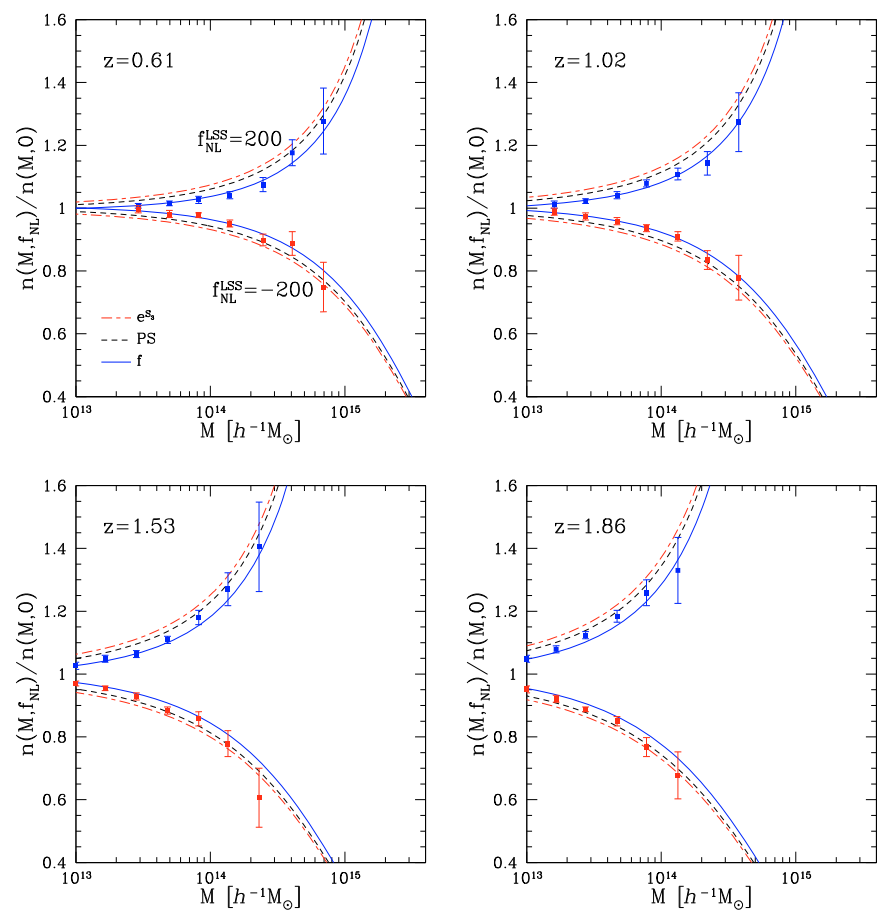

Fig. 2. The ratio $n\left(M, f_{\mathrm{NL}}\right) / n(M, 0)$, of the mass functions obtained for $f_{\mathrm{NL}}^{\mathrm{LSS}}= \pm 200$ over the mass function obtained for Gaussian initial conditions, as a function of $M$ for several redshifts. The dot-dashed line labeled " $\mathrm{e}_{3}$ " is the multiplicative factor (55), the dashed line labeled "PS" is Eq. (58) with the Press-Schechter mass function (44), which also corresponds to the result of Matarrese et al. (2000) (but with $\delta_{\mathrm{L}} \simeq 1.59$ ), the solid line labeled "f" is Eq. (58) with the fitting function (52). The data points are results from the numerical simulations of Grossi et al. (2009).

results in this regime and also agrees with simulations. However, using Eq. (58) with the fitting function (52) appears to agree somewhat better with simulations, especially at low masses. This could be expected from the fact that this procedure ensures that the mass function is properly normalized (in contrast, the simple multiplicative factor (55) is greater than unity and does not reproduce the crossing of both mass functions for $v \sim 1$ ).

On the other hand, let us point out that, contrary to some previous works, we do not need to introduce any ad-hoc parameter $q$ (e.g., through a change of the form $\delta_{\mathrm{c}} \rightarrow \delta_{\mathrm{c}} \sqrt{q}$ as in Grossi et al. 2009) to obtain a good match with numerical simulations. This decrease in the linear threshold with respect to the standard value $\delta_{\mathrm{c}} \simeq 1.6754$ (for $\Omega_{\mathrm{m} 0}=0.27$ ) is actually obtained in our approach by using the exact linear threshold $\delta_{\mathrm{L}}=\mathcal{F}^{-1}(200) \simeq 1.59$, as explained above. Therefore, the advantage of this procedure is that we do not need to run new simulations for other cosmologies to obtain a fit for such a $q$-factor, since the value $\delta_{\mathrm{L}}=\mathcal{F}^{-1}(200)$ can always be computed from the spherical collapse dynamics.

Next, we compare our results with numerical simulations from Dalal et al. (2008) in Fig. 3. We again show the ratio $n\left(M, f_{\mathrm{NL}}\right) / n(M, 0)$ as a function of mass, but for a greater primordial non-Gaussianity, $f_{\mathrm{NL}}= \pm 500$. For $f_{\mathrm{NL}}=500$ (upper panel), we note as in Dalal et al. (2008) that the prediction of (58) computed with the Press-Schechter mass function (44) (i.e. the result of Matarrese et al. 2000, but with $\delta_{\mathrm{L}} \simeq 1.59$ ) tends to overestimate the deviations from the Gaussian case (and the simple multiplicative factor (55) fares somewhat worse). However, using Eq. (58) with the correct Gaussian mass function (52) decreases this ratio somewhat (as in Fig. 2) and provides good 
P. Valageas: Mass function and bias of dark matter halos for non-Gaussian initial conditions
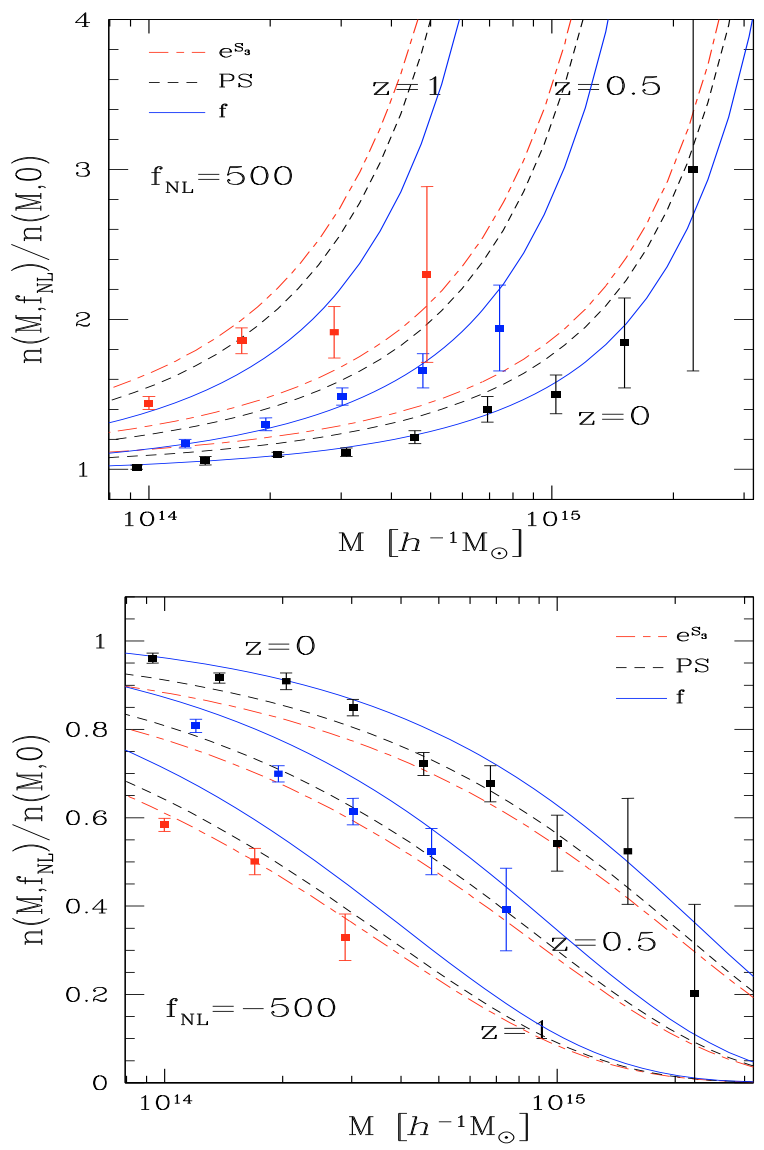

Fig. 3. Same as Fig. 2, but with $f_{\mathrm{NL}}=500$ (upper panel) and $f_{\mathrm{NL}}=$ -500 (lower panel). The data points are the results of the numerical simulations of Dalal et al. (2008).

agreement with the simulations. For $f_{\mathrm{NL}}=-500$ (lower panel) the match is not as good. However, in that case the agreement might improve at higher masses, where there are no data points but where the predictions (55) or (58) are asymptotically exact. Moreover, the discrepancy between theoretical predictions and numerical simulations has the same order as the deviation between the different theoretical curves. Since the latter show the same exact high-mass behavior (at the leading order given by the exponential cutoff (44)), these deviations show the sensitivity of the mass functions to the details of the theoretical prescriptions. These power-law prefactors have not been rigorously derived (and are expected not to be exact for all formulae used here). Therefore, the deviation between these theoretical predictions estimates the theoretical uncertainty for the ratio of the halo mass functions. Then, taking this theoretical uncertainty into account, we can see that the agreement with the numerical results is still reasonable. For practical purposes, when one tries to derive constraints on cosmology from observations of halo mass functions, it would be useful to consider several theoretical prescriptions in addition to the best prediction (58)-(52), as in Figs. 2 and 3 , so as to take the theoretical uncertainty into account in the analysis.

\section{Bias of dark matter halos}

\subsection{Two-cell saddle point and real-space bias}

We now consider the bias of dark matter halos, or more precisely their two-point correlation function. As in Valageas (2009b), following Kaiser (1984), we identify rare massive halos with positive density fluctuations in the linear density field. Thus, we first consider the bivariate probability distribution, $\mathcal{P}_{\mathrm{L}}\left(\delta_{\mathrm{L} 1}, \delta_{\mathrm{L} 2}\right)$, of the linear density contrasts $\delta_{\mathrm{L} 1}$ and $\delta_{\mathrm{L} 2}$ within two spheres $V_{1}$ and $V_{2}$, of radii $q_{1}$ and $q_{2}$, and separated by the distance $s$. Note that we distinguish the Lagrangian distance $s$ between the halos measured in the linear density field from their Eulerian distance $x$ measured in the nonlinear density field. Indeed, since halos have moved through their mutual gravitational attraction, these two distances are usually different. Proceeding as in Sect. 3.1 to obtain the rare-event tails,

$\mathcal{P}_{\mathrm{L}}\left(\delta_{\mathrm{L} 1}, \delta_{\mathrm{L} 2}\right) \sim \max _{\left\{\chi[\boldsymbol{q}] \mid \delta_{\mathrm{L} q 1}=\delta_{\mathrm{L} 1}, \delta_{\mathrm{L} q 2}=\delta_{\mathrm{L} 2}\right\}} \mathrm{e}^{-\frac{1}{2} \chi \cdot C_{\mathrm{L}}^{-1} \cdot \chi}$,

we are led to introduce the action

$$
\begin{aligned}
\mathcal{S}\left[\chi, \lambda_{1}, \lambda_{2}\right]= & \lambda_{1}\left(\delta_{\mathrm{L} 1}-\delta_{\mathrm{L} q 1}[\chi]\right)+\lambda_{2}\left(\delta_{\mathrm{L} 2}-\delta_{\mathrm{L} q 2}[\chi]\right) \\
& +\frac{1}{2} \chi \cdot C_{\mathrm{L}}^{-1} \cdot \chi,
\end{aligned}
$$

which now involves the two Lagrange multipliers $\lambda_{1}$, and $\lambda_{2}$. We again obtain the minimizer of the Gaussian weight $\Gamma_{12}=$ $\left(\chi \cdot C_{\mathrm{L}}^{-1} \cdot \chi\right) / 2$ of Eq. (60) by differentiating the action $\mathcal{S}$ with respect to $\chi, \lambda_{1}$ and $\lambda_{2}$, and we solve these equations as a perturbative series over $f_{\mathrm{NL}}$. At order zero, we recover the Gaussian terms

$$
\begin{aligned}
& \chi^{(0)}(\boldsymbol{q})=\lambda_{1}^{(0)} \int_{V_{1}} \frac{\mathrm{d} \boldsymbol{q}_{1}}{V_{1}} C_{\mathrm{L}}\left(\boldsymbol{q}, \boldsymbol{q}_{1}\right)+\lambda_{2}^{(0)} \int_{V_{2}} \frac{\mathrm{d} \boldsymbol{q}_{2}}{V_{2}} C_{\mathrm{L}}\left(\boldsymbol{q}, \boldsymbol{q}_{2}\right) \\
& \lambda_{1}^{(0)}=\frac{\sigma_{2}^{2} \delta_{\mathrm{L} 1}-\sigma_{12}^{2} \delta_{\mathrm{L} 2}}{\sigma_{1}^{2} \sigma_{2}^{2}-\sigma_{12}^{4}} \\
& \lambda_{2}^{(0)}=\frac{\sigma_{1}^{2} \delta_{\mathrm{L} 2}-\sigma_{12}^{2} \delta_{\mathrm{L} 1}}{\sigma_{1}^{2} \sigma_{2}^{2}-\sigma_{12}^{4}}
\end{aligned}
$$

where we note $\sigma_{i}^{2}=\sigma_{q_{i}, q_{i}}^{2}(0)$ and $\sigma_{12}^{2}=\sigma_{q_{1}, q_{2}}^{2}(s)$ from Eq. (15). At first order we obtain

$$
\begin{aligned}
& \chi^{(1)}(\boldsymbol{q})=\lambda_{1}^{(1)} \int_{V_{1}} \frac{\mathrm{d} \boldsymbol{q}_{1}}{V_{1}} C_{\mathrm{L}}\left(\boldsymbol{q}, \boldsymbol{q}_{1}\right)+\lambda_{2}^{(1)} \int_{V_{2}} \frac{\mathrm{d} \boldsymbol{q}_{2}}{V_{2}} C_{\mathrm{L}}\left(\boldsymbol{q}, \boldsymbol{q}_{2}\right) \\
& +2 \lambda_{1}^{(0)} \int_{V_{1}} \frac{\mathrm{d} \boldsymbol{q}_{1}}{V_{1}} \int \mathrm{d} \boldsymbol{q}_{1}^{\prime} \mathrm{d} \boldsymbol{q}_{2}^{\prime} f_{\mathrm{NL}}^{\delta}\left(\boldsymbol{q}_{1} ; \boldsymbol{q}_{1}^{\prime}, \boldsymbol{q}_{2}^{\prime}\right) C_{\mathrm{L}}\left(\boldsymbol{q}, \boldsymbol{q}_{1}^{\prime}\right) \chi^{(0)}\left(\boldsymbol{q}_{2}^{\prime}\right) \\
& +2 \lambda_{2}^{(0)} \int_{V_{2}} \frac{\mathrm{d} \boldsymbol{q}_{2}}{V_{2}} \int \mathrm{d} \boldsymbol{q}_{1}^{\prime} \mathrm{d} \boldsymbol{q}_{2}^{\prime} f_{\mathrm{NL}}^{\delta}\left(\boldsymbol{q}_{2} ; \boldsymbol{q}_{1}^{\prime}, \boldsymbol{q}_{2}^{\prime}\right) C_{\mathrm{L}}\left(\boldsymbol{q}, \boldsymbol{q}_{1}^{\prime}\right) \chi^{(0)}\left(\boldsymbol{q}_{2}^{\prime}\right)
\end{aligned}
$$

$$
\begin{aligned}
\lambda_{1}^{(1)}\left(\sigma_{1}^{2} \sigma_{2}^{2}\right. & \left.-\sigma_{12}^{4}\right)=\lambda_{1}^{(0) 2}\left[\sigma_{12}^{2}\left(2 f_{1 ; 12}+f_{2 ; 11}\right)-3 \sigma_{2}^{2} f_{1 ; 11}\right] \\
& +2 \lambda_{1}^{(0)} \lambda_{2}^{(0)}\left[\sigma_{12}^{2}\left(2 f_{2 ; 12}+f_{1 ; 22}\right)-\sigma_{2}^{2}\left(2 f_{1 ; 12}+f_{2 ; 11}\right)\right] \\
+ & \lambda_{2}^{(0) 2}\left[3 \sigma_{12}^{2} f_{2 ; 22}-\sigma_{2}^{2}\left(2 f_{2 ; 12}+f_{1 ; 22}\right)\right]
\end{aligned}
$$

and

$$
\begin{aligned}
& \lambda_{2}^{(1)}\left(\sigma_{1}^{2} \sigma_{2}^{2}-\sigma_{12}^{4}\right)=\lambda_{1}^{(0) 2}\left[3 \sigma_{12}^{2} f_{1 ; 11}-\sigma_{1}^{2}\left(2 f_{1 ; 12}+f_{2 ; 11}\right)\right] \\
& +2 \lambda_{1}^{(0)} \lambda_{2}^{(0)}\left[\sigma_{12}^{2}\left(2 f_{1 ; 12}+f_{2 ; 11}\right)-\sigma_{1}^{2}\left(2 f_{2 ; 12}+f_{1 ; 22}\right)\right] \\
& \quad+\lambda_{2}^{(0) 2}\left[\sigma_{12}^{2}\left(2 f_{2 ; 12}+f_{1 ; 22}\right)-3 \sigma_{1}^{2} f_{2 ; 22}\right]
\end{aligned}
$$

where we note for instance $f_{1 ; 22}=f_{a ; b b}(s)$ and $f_{2 ; 12}=f_{b ; a b}(s)$, with $V_{a}=V_{1}, V_{b}=V_{2}$, from Eqs. (19), (20). Next, the Gaussian weight $\Gamma_{12}=\left(\chi \cdot C_{\mathrm{L}}^{-1} \cdot \chi\right) / 2$ reads at order zero

$\Gamma_{12}^{(0)}=\frac{\sigma_{2}^{2} \delta_{\mathrm{L} 1}^{2}+\sigma_{1}^{2} \delta_{\mathrm{L} 2}^{2}-2 \sigma_{12}^{2} \delta_{\mathrm{L} 1} \delta_{\mathrm{L} 2}}{2\left(\sigma_{1}^{2} \sigma_{2}^{2}-\sigma_{12}^{4}\right)}$, 
and at first order,

$$
\begin{aligned}
\Gamma_{12}^{(1)}= & \lambda_{1}^{(1)}\left(\sigma_{1}^{2} \lambda_{1}^{(0)}+\sigma_{12}^{2} \lambda_{2}^{(0)}\right)+\lambda_{2}^{(1)}\left(\sigma_{12}^{2} \lambda_{1}^{(0)}+\sigma_{2}^{2} \lambda_{2}^{(0)}\right) \\
& +2 \lambda_{1}^{(0) 3} f_{1 ; 11}+2 \lambda_{1}^{(0) 2} \lambda_{2}^{(0)}\left(2 f_{1 ; 12}+f_{2 ; 11}\right) \\
& +2 \lambda_{1}^{(0)} \lambda_{2}^{(0) 2}\left(2 f_{2 ; 12}+f_{1 ; 22}\right)+2 \lambda_{2}^{(0) 3} f_{2 ; 22} .
\end{aligned}
$$

Therefore, in the rare-event limit the tail of the bivariate distribution (60) reads as

$\mathcal{P}_{\mathrm{L}}\left(\delta_{\mathrm{L} 1}, \delta_{\mathrm{L} 2}\right) \sim \mathcal{P}_{\mathrm{L}}\left(\delta_{\mathrm{L} 1}\right) \mathcal{P}_{\mathrm{L}}\left(\delta_{\mathrm{L} 2}\right) \mathrm{e}^{-\Delta \Gamma}$,

with

$\Delta \Gamma=\Gamma_{12}-\Gamma_{1}-\Gamma_{2}$,

where $\Gamma_{12}$ is given by Eqs. (68), (69) and $\Gamma_{1}$ and $\Gamma_{2}$ by Eqs. (42), (43) for each sphere $V_{1}$ and $V_{2}$. Next, following Valageas (2009b), we write the halo two-point correlation as

$1+\xi_{M_{1}, M_{2}}(x)=\left(1+\delta_{\mathrm{L} M}(s)\right) \mathrm{e}^{-\Delta \Gamma}$,

where the factor $\left(1+\delta_{\mathrm{L} M}(s)\right)$ models the effects associated with the mapping from Lagrangian to Eulerian space. This is the local linear density contrast at radius $s$ from a halo of mass $M=\max \left(M_{1}, M_{2}\right)$ (to keep the symmetry $M_{1} \leftrightarrow M_{2}$ ), as given by Eqs. (39), (40). A sufficiently accurate approximation would be to use only the zeroth-order term (39), as shown by Fig. 1, but taking the correction (40) into account brings no further difficulty. Here we approximated the nonlinear density contrast $\delta_{M}$ by the linear density contrast $\delta_{\mathrm{LM}}$, since at a large distance where $\delta_{M} \ll 1$ we have $\delta_{M} \simeq \delta_{\mathrm{L} M}$. Next, we must express the Lagrangian separation $s$ in terms of the Eulerian distance $x$. Following Valageas (2009b), at the lowest order where we consider each halo as a test particle that falls into the potential well built by the other halo, we obtain $s$ as the solution of the implicit equation

$x=s\left(1-\frac{\delta_{\mathrm{L} M_{1}}(s)}{3}-\frac{\delta_{\mathrm{L} M_{2}}(s)}{3}\right)$,

where $\delta_{\mathrm{L} M_{i}}(s)$ is the linear density contrast within radius $s$ of the halo of mass $M_{i}$, given by Eqs. (36), (37). At a large separation, this relation can be inverted as

$s=x\left(1+\frac{\delta_{\mathrm{L} M_{1}}(x)}{3}+\frac{\delta_{\mathrm{L} M_{2}}(x)}{3}\right)$,

which provides an explicit expression for $s$.

Finally, we define the real-space halo bias as the ratio of the halo and matter two-point correlations,

$b_{M_{1}, M_{2}}^{2}(x)=\frac{\xi_{M_{1}, M_{2}}(x)}{\xi(x)}$.

Since at a large distance the matter correlation is within the linear regime, $\xi(x) \simeq \sigma_{0,0}^{2}(x)$, we also write in this limit

$b_{M_{1}, M_{2}}^{2}(x) \simeq \frac{\xi_{M_{1}, M_{2}}(x)}{\sigma_{0,0}^{2}(x)}$,

which fully determines the halo bias from Eq. (72).

For equal-mass halos of radius $q$, defined by the same threshold $\delta_{\mathrm{L}}$, the two Lagrange multipliers are equal, $\lambda_{1}=\lambda_{2}=\lambda$, and Eqs. $(63,64)$ and $(66,67)$ simplify as

$\lambda^{(0)}=\frac{\delta_{\mathrm{L}}}{\sigma^{2}+\sigma_{12}^{2}}$, and

$\lambda^{(1)}=\frac{-3 \delta_{\mathrm{L}}^{2}}{\left(\sigma^{2}+\sigma_{12}^{2}\right)^{3}}\left(f_{2 ; 11}+2 f_{1 ; 12}+f_{1 ; 11}\right)$,

where we note $\sigma^{2}=\sigma_{q}^{2}$. This yields, for the Gaussian weight $\Gamma_{12}$,

$\Gamma_{12}^{(0)}=\frac{\delta_{\mathrm{L}}^{2}}{\sigma^{2}+\sigma_{12}^{2}}$,

and

$\Gamma_{12}^{(1)}=\frac{-2 \delta_{\mathrm{L}}^{3}}{\left(\sigma^{2}+\sigma_{12}^{2}\right)^{3}}\left(f_{2 ; 11}+2 f_{1 ; 12}+f_{1 ; 11}\right)$.

Then, the difference $\Delta \Gamma$ defined in Eq. (71), which measures the correlation between rare events in the linear density field, writes as

$\Delta \Gamma^{(0)}=\frac{-\sigma_{12}^{2} \delta_{\mathrm{L}}^{2}}{\sigma^{2}\left(\sigma^{2}+\sigma_{12}^{2}\right)}$,

and

$\Delta \Gamma^{(1)}=\frac{-2 \delta_{\mathrm{L}}^{3}}{\left(\sigma^{2}+\sigma_{12}^{2}\right)^{3}}\left[f_{2 ; 11}+2 f_{1 ; 12}+\frac{\sigma^{6}-\left(\sigma^{2}+\sigma_{12}^{2}\right)^{3}}{\sigma^{6}} f_{1 ; 11}\right]$.

We can check that $\Delta \Gamma$ vanishes for $s \rightarrow \infty$, since all mixed quantities, $\sigma_{12}^{2}, f_{1 ; 12}$ and $f_{2 ; 11}$, go to zero.

As stressed in Politzer \& Wise (1984), real-space formulae such as $(72)$, which are obtained in the rare event $\left(\delta_{\mathrm{L}} / \sigma \gg 1\right)$ and large separation $\left(\sigma_{12} \ll \sigma\right)$ limits, do not assume that the exponent $\Delta \Gamma$ is small. In fact, as shown in Valageas (2009b), at high redshift one can probe a regime where this exponent is large, so that one needs to keep the nonlinear form (72), which yields a nonlinear bias. As seen from Eq. (81), this regime corresponds to very massive halos, $\delta_{\mathrm{L}} / \sigma \rightarrow \infty$, at fixed (low) ratio $\sigma_{12} / \sigma$. Nevertheless, in the regime where $\Delta \Gamma$ is small (i.e. in the large separation limit $x \rightarrow \infty$ ), which covers the cases of interest encountered at low redshift, we can expand the exponential in Eq. (72). Then, at the lowest order over the terms that vanish in the large separation limit, we obtain

$x \rightarrow \infty: \quad \xi_{M}(x) \simeq \delta_{\mathrm{L} M}(s)-\Delta \Gamma$

for equal-mass halos, whence

$$
\begin{array}{r}
\xi_{M}(x) \simeq \frac{\delta_{\mathrm{L}}}{\sigma_{q}^{2}} \sigma_{q, 0}^{2}(s)+\frac{\delta_{\mathrm{L}}^{2}}{\sigma_{q}^{4}}\left[f_{0 ; q q}(s)+2 f_{q ; 0 q}(s)-3 \frac{f_{q ; q q}}{\sigma_{q}^{2}} \sigma_{q, 0}^{2}(s)\right] \\
+\frac{\delta_{\mathrm{L}}^{2}}{\sigma_{q}^{4}} \sigma_{q, q}^{2}(s)+2 \frac{\delta_{\mathrm{L}}^{3}}{\sigma_{q}^{6}}\left[f_{2 ; 11}(s)+2 f_{1 ; 12}(s)-3 \frac{f_{1 ; 11}}{\sigma_{q}^{2}} \sigma_{q, q}^{2}(s)\right] .
\end{array}
$$

Hereafter we call the bias $b_{M}^{2}(x)$ obtained from Eqs. (84) and (76) the "linearized" bias.

Following the approach of Kaiser (1984), Matarrese \& Verde (2008) also computed the effect of local-type nonGaussianity (1) on the halo two-point correlation. Drawing on earlier work by Matarrese et al. (1986), who computed the $n$-point halo correlations by expanding the relevant pathintegrals and next resumming the series within a large-distance and rare-event approximation, they obtained expressions of the form (72) without the prefactor $\left(1+\delta_{\mathrm{L} M}\right)$ and (84) without the terms in the first line, which arise from this prefactor, and the last term $f_{1 ; 11}$ in the second bracket. This term arises from the factor $\sigma_{12}^{2}$ in the denominator of expression (80), associated with 
P. Valageas: Mass function and bias of dark matter halos for non-Gaussian initial conditions

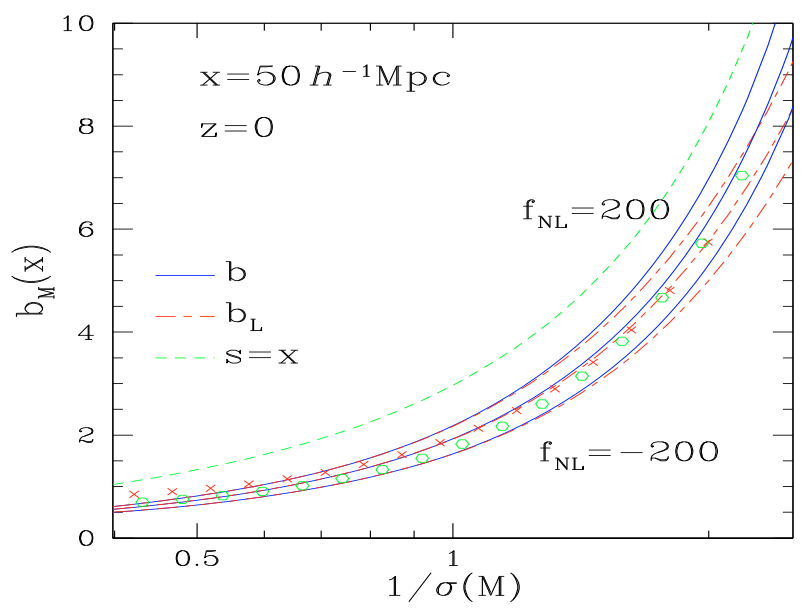

Fig. 4. The halo bias $b_{M}(x)$, as a function of $\sigma(M)$, at fixed redshift $z=0$ and distance $x=50 h^{-1}$ Mpc. The solid lines " $b$ " are the nonlinear theoretical prediction of Eqs. (72), (73) and (76), for $f_{\mathrm{NL}}= \pm 200$ and $f_{\mathrm{NL}}=0$ (i.e. Gaussian case, intermediate line), while the dot-dashed lines " $b_{\mathrm{L}}$ " are the linearized bias of Eq. (84). The upper dashed line " $s=x$ " shows the result obtained in the Gaussian case by setting $s=x$ in Eq. (72). The points are the fits to Gaussian numerical simulations, from Sheth et al. (2001) (crosses) and Pillepich (2010) (circles).

the effect of primordial non-Gaussianity on the one-point distribution (43). It can be seen as a renormalization of the Gaussian term $\left(\delta_{\mathrm{L}}^{2} / \sigma_{q}^{4}\right) \sigma_{q, q}^{2}(s)$, since it shows the same scale dependence through the function $\sigma_{q, q}^{2}(s)$. The presence of such a term has already been noticed in Slosar et al. (2008) in Fourier space, and Desjacques et al. (2009) points out that it needs to be included to obtain good agreement with numerical simulations. This will give rise to the last term in Eq. (85) and the second term in Eqs. (91) and (93) below. On the other hand, the terms in the first line of Eq. (84), associated with the prefactor $\left(1+\delta_{\mathrm{LM}}\right)$ in Eq. (72), stem from the mapping from Lagrangian to Eulerian space, and the first bracket in Eq. (84) expresses the (weak) effect of primordial non-Gaussianity on this mapping. Another difference between Eqs. (72) and (84) and previous works is that (within some approximation) we pay attention to the difference between Lagrangian and Eulerian distances $s$ and $x$. This can play a non-negligible role as seen in Fig. 4 below.

We show in Fig. 4 the halo bias $b_{M}(x)$ as a function of $\sigma(M)$ at fixed distance $x=50 h^{-1} \mathrm{Mpc}$ and redshift $z=0$, for $f_{\mathrm{NL}}= \pm 200$ and $f_{\mathrm{NL}}=0$. We display both the nonlinear result of Eq. (72) and the linear result of Eq. (84). In addition, for the Gaussian case we also display the bias obtained by setting $s=x$ in Eq. (72). As in Valageas (2009b), for the Gaussian case $\left(f_{\mathrm{NL}}=0\right)$ we obtain good agreement with the fits to numerical simulations of Sheth et al. (2001) and Pillepich (2010). Moreover, we can see that it is important to take the displacement of the halos into account through Eq. (73), as the approximation $s=x$ significantly overestimates the bias. We checked that using the simpler Eq. (74) gives a close result to the one obtained with Eq. (73) and also agrees with the simulations. Thus, for practical purposes it is sufficient to use Eq. (74). We can see that for all cases shown in Fig. 4 the linear bias from Eq. (84) gives results that are very close to the fully nonlinear expression (72). This justifies the use of such linearized expressions in this regime. This will be especially useful in Sect. 4.2, where we consider the bias of dark matter halos in Fourier space. Indeed, it is easier to take the Fourier transform of Eq. (84), which allows us to recover the results obtained in previous works. Thus, one
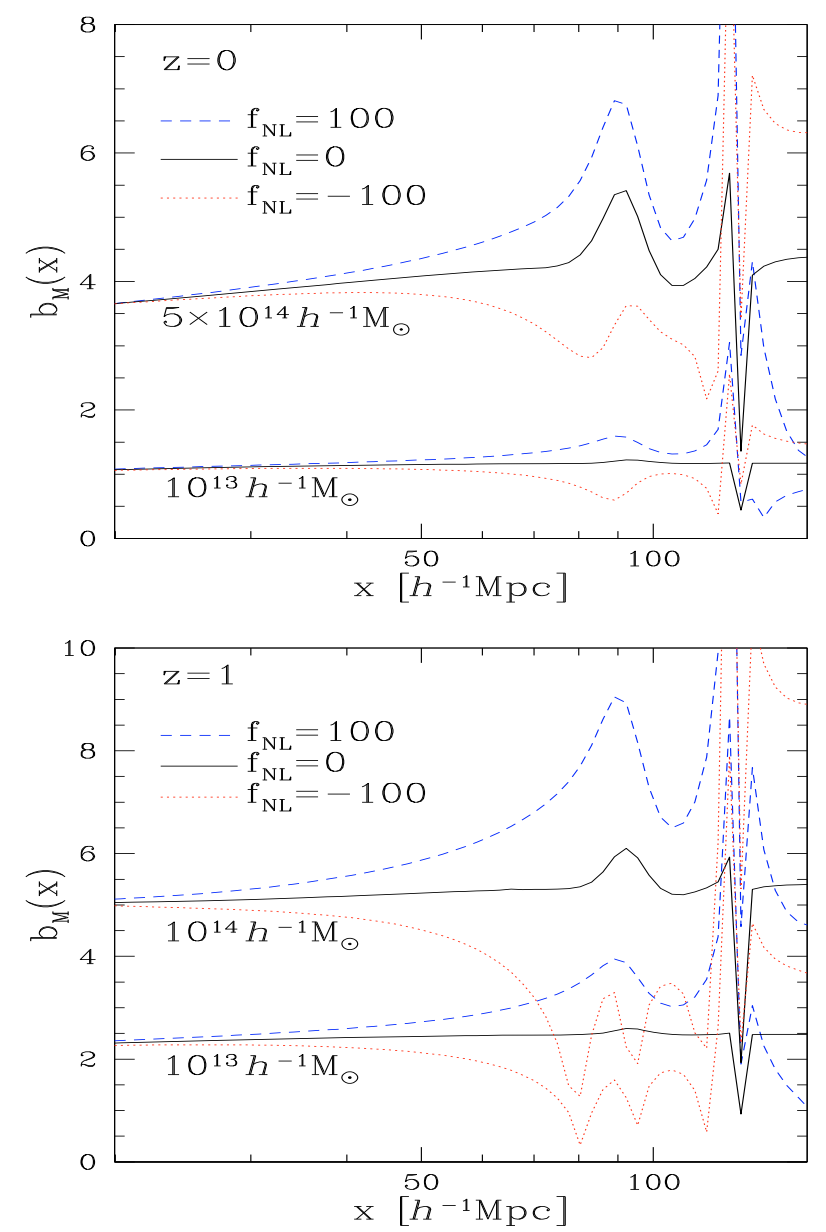

Fig. 5. The halo bias $b_{M}(x)$ as a function of distance $x$, at redshifts $z=0$ (upper panel) and $z=1$ (lower panel) for several masses. We show the cases $f_{\mathrm{NL}}=0$ (solid lines), $f_{\mathrm{NL}}=100$ (dashed lines), and $f_{\mathrm{NL}}=-100$ (dotted lines). The divergences at $x \sim 120 h^{-1} \mathrm{Mpc}$ come from the halo and matter correlations not changing sign at the same distance.

interest of the real-space results (72)-(84) is to provide a check on whether linearized predictions (i.e. where the halo correlation only involves the matter power spectrum at linear order) are valid. Then, in agreement with those studies (which were mostly performed in Fourier space and led to Eq. (2), see Dalal et al. 2008; Slosar et al. 2008), and with Eq. (84), we can see that the deviation from the Gaussian bias, $b_{M}\left(x, f_{\mathrm{NL}}\right)-b_{M}(x, 0)$, grows linearly with $b_{M}(x, 0)$ at large bias and has the same sign as $f_{\mathrm{NL}}$.

Next, we display in Fig. 5 the dependence on the distance $x$ of the bias obtained for several masses at redshifts $z=0$ and $z=1$. More precisely, we show the ratio $\sqrt{\left|\xi_{M}(x) / \xi(x)\right|}$, since the halo and matter correlations do not change sign at the same point. We plot the cases $f_{\mathrm{NL}}= \pm 100$, as well as the Gaussian case $f_{\mathrm{NL}}=0$. While the Gaussian bias is roughly constant on large scales, up to $\sim 100 \mathrm{~h}^{-1} \mathrm{Mpc}$ (in agreement with previous studies, Mo \& White 1996; Mo et al. 1997), the non-Gaussian bias shows a strong scale dependence, with a deviation from the Gaussian bias that roughly grows as $x^{2}$ up to $\sim 100 h^{-1} \mathrm{Mpc}$. This agrees with the $k^{-2}$ behavior observed in Fourier space, see Eq. (2) above (Dalal et al. 2008; Slosar et al. 2008) and Eq. (93) below.

To see the scaling of the real-space correction $\Delta b_{M}\left(x, f_{\mathrm{NL}}\right)=$ $b_{M}\left(x, f_{\mathrm{NL}}\right)-b_{M}(x, 0)$ to the Gaussian bias more clearly, we show in Fig. 6 the ratio $\left[\Delta b_{M}\left(x, f_{\mathrm{NL}}\right) / b_{M}(x, 0)\right] / x_{50}^{2}$, with $x_{50}=$ $x /\left(50 h^{-1} \mathrm{Mpc}\right)$. We display the results obtained from Eq. (72) 

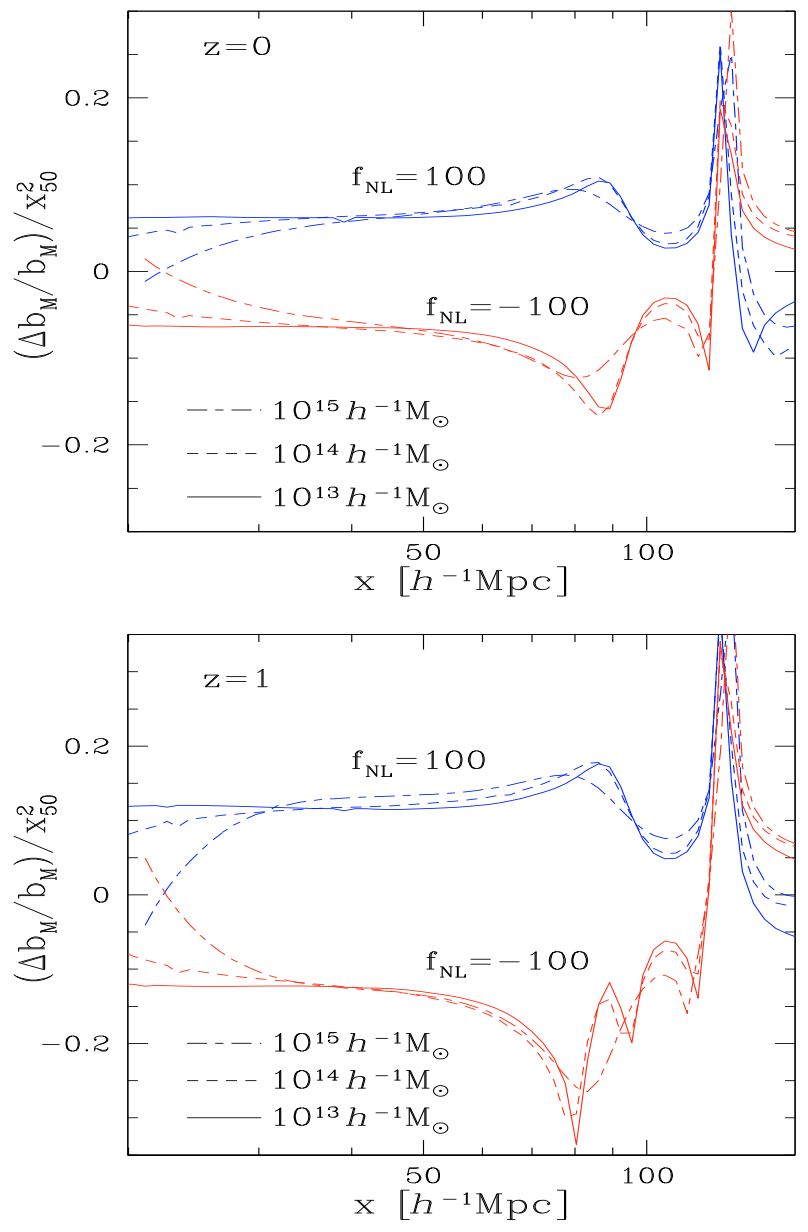

Fig. 6. The real-space ratio $\left[\Delta b_{M}\left(x, f_{\mathrm{NL}}\right) / b_{M}(x, 0)\right] / x_{50}^{2}$ of the correction $\Delta b_{M}\left(x, f_{\mathrm{NL}}\right)=b_{M}\left(x, f_{\mathrm{NL}}\right)-b_{M}(x, 0)$ to the Gaussian bias $b_{M}(x, 0)$, divided by the factor $x_{50}^{2}$ with $x_{50}=x /\left(50 h^{-1} \mathrm{Mpc}\right)$, as a function of distance $x$. We show the cases $f_{\mathrm{NL}}=100$ (upper lines) and $f_{\mathrm{NL}}=-100$ (lower lines) for several masses at redshifts $z=0$ (upper panel) and $z=1$ (lower panel), from Eq. (72).

for several masses at $z=0$ (upper panel) and $z=1$ (lower panel) for $f_{\mathrm{NL}}= \pm 100$. We can see that over the range $30<$ $x<90 h^{-1} \mathrm{Mpc}$ all curves roughly collapse onto one another. This means that the real-space correction roughly scales as $\Delta b_{M}(x) \propto f_{\mathrm{NL}} b_{M}(x, 0) x^{2}$ over this range, which roughly agrees with the Fourier-space scaling (2) (here we neglected any constant offset, such as the factor -1 in Eq. (2), see the discussion of Eq. (94) below). It appears that our predictions scale more closely as $x^{2}$, as shown in Fig. 6, than as $\alpha(1 / x)^{-1}$, which would be suggested by Eqs. (2), (93) (at these scales the transfer function already deviates from unity). This agrees with the behavior observed in Fourier space in Fig. 10 below. The masses shown in Fig. 6 span the range $1.2<b_{M}(x, 0)<5.9$ at $x=50 h^{-1} \mathrm{Mpc}$ and $z=0$, and $2.4<b_{M}(x, 0)<15.4$ at $z=1$, so that the linear scaling with $b_{M}(x, 0)$ of the correction $\Delta b_{M}\left(x, f_{\mathrm{NL}}\right)$ appears to be a good approximation.

Below $30 h^{-1}$ Mpc higher masses show steeper scale dependence for $\Delta b_{M}\left(x, f_{\mathrm{NL}}\right)$. At very large distance, $x>100 h^{-1} \mathrm{Mpc}$, the oscillations seen in Figs. 5 and 6 are caused by the baryon acoustic oscillation. Indeed, the baryon oscillations seen in the halo and matter two-point correlations are not exactly proportional, since the halo correlation is not exactly proportional to $\sigma_{0,0}^{2}(x)$, even in the Gaussian case and in the linear regime (for instance it involves the smoothing scale $q$, see Eq. (84)).
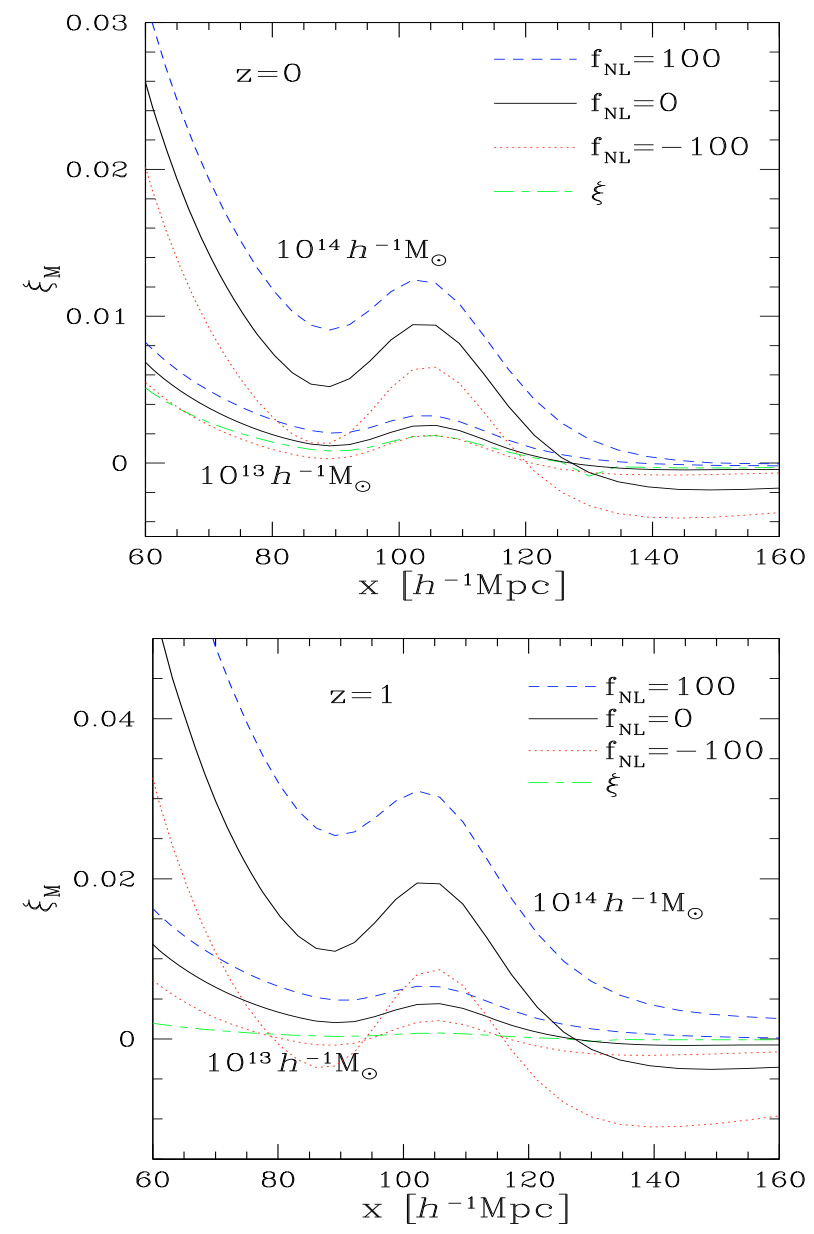

Fig. 7. The halo $\left(f_{\mathrm{NL}}=0, \pm 100\right)$ and matter $(\xi)$ two-point correlations at redshifts $z=0$ (upper panel) and $z=1$ (lower panel). We show the curves obtained for the masses $M=10^{13}$ and $10^{14} h^{-1} M_{\odot}$.

This yields the non-monotonic behavior seen in Figs. 5 and 6 around $100 \mathrm{~h}^{-1} \mathrm{Mpc}$. For the same reason, the halo and matter correlations do not exactly vanish at the same distance, which gives rise to the divergent spike at $\sim 120 h^{-1} \mathrm{Mpc}$. These features simply mean that it is no longer useful to work with the bias $b_{M}$ on these scales, which only makes sense if the halo and matter correlations are roughly proportional. In this range, where the correlations show some oscillations and change sign, it is no longer a good approximation to write the halo correlation in terms of the matter correlation multiplied by some slowly varying bias factor. Then, one instead needs to directly study the halo and matter correlations themselves.

Thus, we compare in Fig. 7 the halo and matter two-point correlations. We focus on large scales to see how the baryon acoustic oscillation is modified when one uses massive halos as a tracer of the initial matter power spectrum. In agreement with previous works (Desjacques 2008), we can see that the oscillation is strongly amplified for massive halos that have a strong bias. This amplification still holds for significant primordial non-Gaussianity $\left(f_{\mathrm{NL}}= \pm 100\right)$, although it appears to be slightly lower for positive $f_{\mathrm{NL}}$. Moreover, the peak of the oscillation shows no significant shift, so that a measure of its position appears to be a robust ruler for constraining cosmology, independently of the halo bias and of the primordial nonGaussianity. In contrast, the distance at which the two-point correlation changes sign is not significantly modified as one goes from the matter to the halo correlation in the Gaussian case, but 
it is fairly sensitive to the primordial non-Gaussianity. In particular, a positive $f_{\mathrm{NL}}$ shifts this point to a greater distance. However, theoretical and observational error bars may be too large to use this effect to constrain $f_{\mathrm{NL}}$ in a competitive manner compared to other probes.

\subsection{Fourier-space bias}

Rather than the real-space two-point correlation, recent works have mostly studied the effect of primordial non-Gaussianity on the halo power spectrum, where at lowest order the Poisson Eq. (3) directly gives an estimate of the form (2) for the deviation from the bias obtained with Gaussian initial conditions (Dalal et al. 2008; Slosar et al. 2008).

It is not convenient to take the Fourier transform of the nonlinear correlation (72), but at moderate redshifts, the linearized form (84) provides a very good approximation. Then, if we also make the approximation $s \simeq x$, which is valid at the lowest order, the Fourier transform of Eq. (84) readily gives the halo power spectrum as

$$
\begin{aligned}
P_{M}(k) \simeq & \frac{\delta_{\mathrm{L}}}{\sigma_{q}^{2}} P_{\mathrm{L}}(k) \tilde{W}(k q)+\frac{\delta_{\mathrm{L}}^{2}}{\sigma_{q}^{4}}\left[\tilde{f}_{0 ; q q}(k)+2 \tilde{f}_{q ; 0 q}(k)\right. \\
& \left.-3 \frac{f_{q ; q q}}{\sigma_{q}^{2}} P_{\mathrm{L}}(k) \tilde{W}(k q)\right]+\frac{\delta_{\mathrm{L}}^{2}}{\sigma_{q}^{4}} P_{\mathrm{L}}(k) \tilde{W}(k q)^{2} \\
& +2 \frac{\delta_{\mathrm{L}}^{3}}{\sigma_{q}^{6}}\left[\tilde{f}_{2 ; 11}(k)+2 \tilde{f}_{1 ; 12}(k)-3 \frac{f_{1 ; 11}}{\sigma_{q}^{2}} P_{\mathrm{L}}(k) \tilde{W}(k q)^{2}\right],
\end{aligned}
$$

where the quantities $\tilde{f}$ are obtained from Eqs. (21), (22). As discussed below Eq. (84), the terms $\tilde{f}_{2 ; 11}(k)$ and $2 \tilde{f}_{1 ; 12}(k)$ in Eq. (85) have already been obtained by Matarrese \& Verde (2008), following Kaiser (1984) by identifying massive halos with rare fluctuations in the linear density field. Defining the Fourier-space bias as (note that this is not the Fourier transform of the realspace bias (75))

$b_{M}^{2}(k)=\frac{P_{M}(k)}{P(k)} \simeq \frac{P_{M}(k)}{P_{\mathrm{L}}(k)}$,

where we used $P(k) \simeq P_{\mathrm{L}}(k)$ at low $k$ for the matter power spectrum, we obtain $b_{M}^{2}(k)$ from Eq. (85). We can also obtain the bias of different-mass halos in a similar fashion, first expanding Eq. (72) and next taking the Fourier transform. To consider the displacement of the halos (i.e. $x \neq s$ ), we can also make the approximation

$P_{M}(k) \rightarrow\left(\frac{x}{s}\right)^{3} P_{M}\left(\frac{x}{s} k\right)$, with $x=\frac{1}{k}$,

where we use the explicit expression (74) for $s(x)$. This expresses that Lagrangian-space wavelengths $\sim s$ (i.e. measured in the linear density field) correspond to smaller Eulerian-space wavelengths $\sim x$ (i.e. measured in the nonlinear density field) because of the displacement of massive halos, which usually have come closer because of their mutual attraction $(x<s)$. This also follows the spirit of the Hamilton et al. (1991) ansatz, translated in Fourier space in Peacock \& Dodds (1996).

For large negative $f_{\mathrm{NL}}$ the halo power spectrum (85) can become negative, because we looked for an expression of the halo correlation, or of the halo power spectrum, at linear order over $f_{\mathrm{NL}}$ as in Eq. (85). However, the power spectrum $P_{M}(k)$ must be positive by definition. Then, in cases where expression (85) turns negative one should consider higher-order terms over
$f_{\mathrm{NL}}$, which would ensure that the power spectrum remains positive. Nevertheless, since such high-order terms are beyond the scope of this article, we consider below the following simple procedure that ensures that $P_{M}(k)$, or the squared bias $b_{M}^{2}(k)$, remain positive. Up to linear order over $f_{\mathrm{NL}}$, the bias (86) reads as

$$
\begin{aligned}
b_{M}\left(k, f_{\mathrm{NL}}\right) & =\sqrt{\frac{P_{M}(k, 0)+\Delta P_{M}\left(k, f_{\mathrm{NL}}\right)}{P(k)}} \\
& \simeq b_{M}(k, 0)\left(1+\frac{1}{2} \frac{\Delta P_{M}\left(k, f_{\mathrm{NL}}\right)}{P_{M}(k, 0)}\right)
\end{aligned}
$$

where $\Delta P_{M}\left(k, f_{\mathrm{NL}}\right)=P_{M}\left(k, f_{\mathrm{NL}}\right)-P_{M}(k, 0)$ is the deviation from the Gaussian halo power spectrum, and we expanded the squareroot. Then, we may use the last expression (88) as the prediction of the halo bias. This amounts to making the transformation

$b_{M}^{2}\left(k, f_{\mathrm{NL}}\right) \rightarrow b_{M}^{2}(k, 0)\left[1+\frac{1}{2}\left(\frac{b_{M}^{2}\left(k, f_{\mathrm{NL}}\right)}{b_{M}^{2}(k, 0)}-1\right)\right]^{2}$,

where the two sides only differ by higher-order terms over $f_{\mathrm{NL}}$ (as $1+\epsilon \simeq(1+\epsilon / 2)^{2}$ up to linear order over $\epsilon$ ) and the right side is always positive.

If we take the limit of very rare events, which is $\sigma_{q} \rightarrow 0$ in Eq. (85), we can only keep the last two terms (note that $\tilde{f} \propto \sigma_{q}^{2}$, see Eq. (92) below),

$$
\begin{aligned}
\sigma_{q} \rightarrow 0: \quad P_{M}(k) \sim & P_{\mathrm{L}}(k) \tilde{W}(k q)^{2}\left[\frac{\delta_{\mathrm{L}}^{2}}{\sigma_{q}^{4}}-6 \frac{\delta_{\mathrm{L}}^{3} f_{1 ; 11}}{\sigma_{q}^{8}}\right] \\
& +2 \frac{\delta_{\mathrm{L}}^{3}}{\sigma_{q}^{6}}\left(\tilde{f}_{2 ; 11}(k)+2 \tilde{f}_{1 ; 12}(k)\right) .
\end{aligned}
$$

At low $k$, with $\tilde{W}(k q) \simeq 1$, and at linear order over $f_{\mathrm{NL}}$, the square root of Eq. (86) gives with Eq. (90) the bias

$$
\begin{aligned}
\sigma_{q} \rightarrow 0, k \rightarrow 0: b_{M}(k) \sim & \frac{\delta_{\mathrm{L}}}{\sigma_{q}^{2}}-3 \frac{\delta_{\mathrm{L}}^{2}}{\sigma_{q}^{6}} f_{1 ; 11} \\
& +\frac{\delta_{\mathrm{L}}^{2}}{\sigma_{q}^{4}} \frac{\tilde{f}_{2 ; 11}(k)+2 \tilde{f}_{1 ; 12}(k)}{P_{\mathrm{L}}(k)} .
\end{aligned}
$$

The first term, $\delta_{\mathrm{L}} / \sigma_{q}^{2}$, is the result obtained by Kaiser (1984) for rare massive halos (i.e. with a strong bias) for Gaussian initial conditions. It is interesting to note that the second term gives a scale-independent correction to the Gaussian bias. This term has already been noticed in Slosar et al. (2008) and Afshordi \& Tolley (2008). As stressed in Desjacques et al. (2009), taking this term into account is required to obtain a good match to numerical simulations. We checked that this is indeed the case to match the numerical results in Figs. 8 and 9 below using Eq. (85). The last two terms depend on the wavenumber $k$. There is an additional term that we neglect in this paper, which arises from the dependence of the matter power spectrum on $f_{\mathrm{NL}}$ (i.e. the denominator in Eq. (86)). However, since this term is much smaller than the other ones, we disregard it here (see Desjacques et al. 2009). For the local non-Gaussianity (7), we find from Eqs. (21), (22)

$$
\begin{aligned}
k \rightarrow 0: \tilde{f}_{2 ; 11}(k) & \sim f_{\mathrm{NL}} \alpha(k) \int \mathrm{d} \boldsymbol{k}_{1} P_{\mathrm{L}}\left(k_{1}\right)^{2} \frac{\tilde{W}\left(k_{1} q\right)^{2}}{\alpha\left(k_{1}\right)^{2}}, \\
\tilde{f}_{1 ; 12}(k) & \sim f_{\mathrm{NL}} \frac{\sigma_{q}^{2} P_{\mathrm{L}}(k)}{\alpha(k)},
\end{aligned}
$$

which yields from Eq. (91)

$$
\sigma_{q} \rightarrow 0, \quad k \rightarrow 0: \quad b_{M}(k) \sim \frac{\delta_{\mathrm{L}}}{\sigma_{q}^{2}}-3 \frac{\delta_{\mathrm{L}}^{2}}{\sigma_{q}^{6}} f_{1 ; 11}+f_{\mathrm{NL}} \frac{2 \delta_{\mathrm{L}}^{2}}{\sigma_{q}^{2} \alpha(k)}
$$



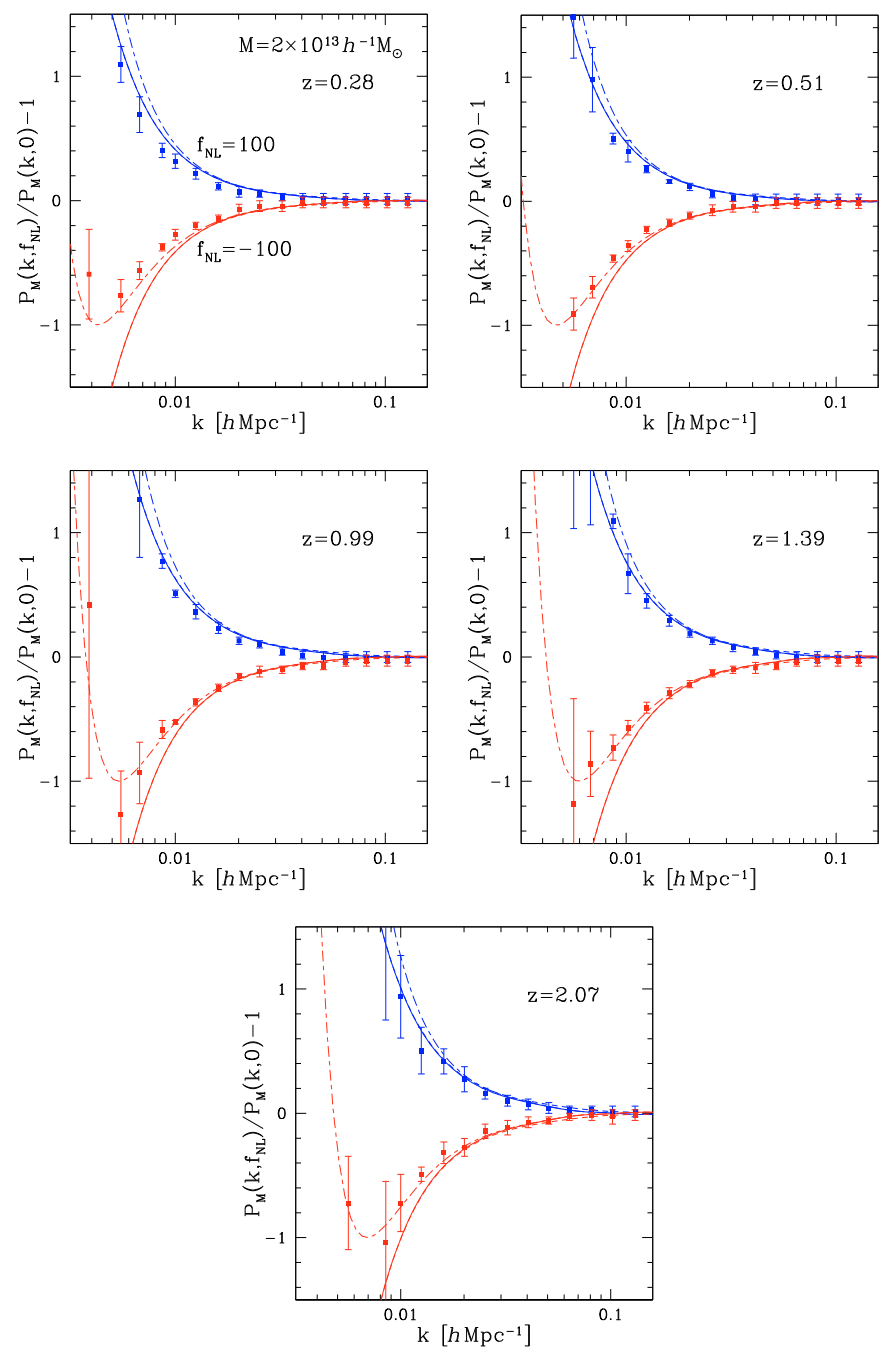

Fig. 8. The correction to the halo power spectrum due to primordial nonGaussianity. We show the ratio $P_{M}\left(k, f_{\mathrm{NL}}\right) / P_{M}(k, 0)-1$ for $f_{\mathrm{NL}}=100$ (upper curves) and $f_{\mathrm{NL}}=-100$ (lower curves). The solid and dashed curves that are almost indistinguishable are Eqs. (85) and (87). The dotdashed curves that are above the solid curves at low $k$ correspond to Eq. (89) which ensures that the halo power spectrum is always positive. The theoretical predictions are for $M=2 \times 10^{13} h^{-1} M_{\odot}$ and the data points are the numerical simulations of Desjacques et al. (2009), for $M>2 \times 10^{13} h^{-1} M_{\odot}$.

Thus, we recover the $k^{-2}$ dependence at low $k$ brought by the local-type non-Gaussianity (1), through the $1 / \alpha(k)$ factor in the last term. The second term in Eq. (93) is the constant shift due to the non-Gaussianity noticed above.

In spite of the $k^{-2}$ dependence at low $k$ obtained in Eq. (93) for the halo bias, the real-space halo two-point correlation is well-defined and finite, as seen in Sect. 4.1. Indeed, Eq. (93) only applies to a limited range, and one cannot write the realspace two-point correlation as a Fourier transform of the form $\xi_{M}(x) \sim \int \mathrm{d} \boldsymbol{k} \mathrm{e}^{-\mathrm{i} k \cdot x}\left[1+f_{\mathrm{NL}} / \alpha(k)\right]^{2} P_{\mathrm{L}}(k)$, which would diverge at low $k$. Thus, the advantage of the real-space approaches, such as the one described in this paper, is that we obtain well-defined results in both real space and Fourier space, and we do not need to regularize integrals by introducing a counterterm associated with a survey-size window, as in Wands \& Slosar (2009). This is reassuring, since one does not expect the halo correlation on a given scale to depend on the size of the survey. Mathematically, the lack of divergence in our approach comes
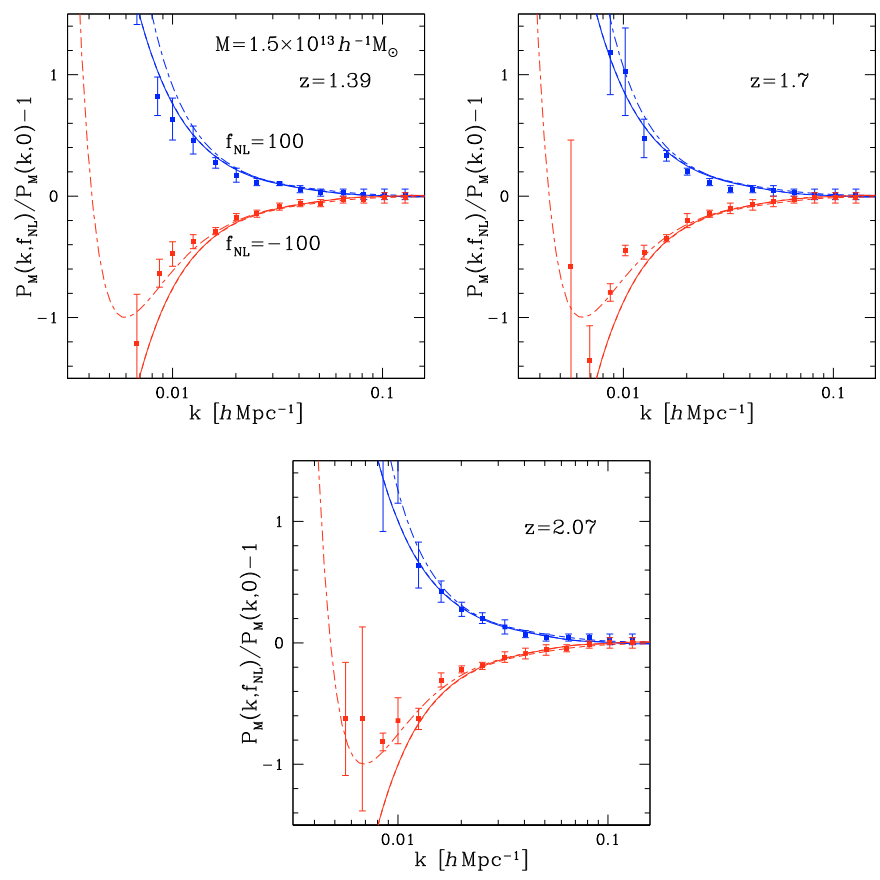

Fig. 9. Same as Fig. 8, but for $M=1.5 \times 10^{13} h^{-1} M_{\odot}$. The data points are the numerical simulations of Desjacques et al. (2009), for $10^{13}<$ $M<2 \times 10^{13} h^{-1} M_{\odot}$.

from the fact that it is the halo power spectrum itself which contains a term of the form $\Delta P_{M}(k) \sim f_{\mathrm{NL}} P_{\mathrm{L}}(k) / \alpha(k)$, see Eqs. (85) and (92), and it is only by expanding the square-root as in (88), $\Delta b=b\left[\sqrt{1+\Delta P_{M} / P_{M}}-1\right] \simeq b \Delta P_{M} /\left(2 P_{M}\right)$, that $b$ can be written as in Eq. (93). As we shall see below, in Figs. 8 and 9, the expression (85) is sufficient to explain the behavior observed in numerical simulations, without introducing worrying divergences.

We compare in Figs. 8 and 9 our results for the Fourier-space bias $b_{M}^{2}(k)$ with numerical simulations from Desjacques et al. (2009). Since the dependence on mass is rather weak, we show our results in Fig. 8 for $M=2 \times 10^{13} h^{-1} M_{\odot}$, whereas the data points are for $M>2 \times 10^{13} h^{-1} M_{\odot}$, and we show our results in Fig. 9 for $M=1.5 \times 10^{13} h^{-1} M_{\odot}$, whereas the data points are for $10^{13}<M<2 \times 10^{13} h^{-1} M_{\odot}$. The predictions (85) and (87) are almost indistinguishable in this regime, and they agree reasonably well with the simulations, except at low $k$ for $f_{\mathrm{NL}}=-100$ where they give a negative halo power spectrum. Equation (89), gives a much better fit to simulations at low $k$ for negative $f_{\mathrm{NL}}$, as could be expected from the fact that it always gives a positive halo power spectrum. However, a priori one should not give too much weight to this improved accuracy in this regime. Indeed, as is clear from Eqs. (88), (89), the solid and dot-dashed curves in Figs. 8, 9 only differ by terms of order $f_{\mathrm{NL}}^{2}$ and beyond. Since all our results have been derived at linear order over $f_{\mathrm{NL}}$, one can expect that Eq. (89) does not include all terms of order $f_{\mathrm{NL}}^{2}$. Then, although for practical purposes it is better to use Eq. (89) in this regime (i.e. negative $f_{\mathrm{NL}}$ at low $k$ ), it is still useful to also consider Eq. (85), as the deviation between both predictions should give an estimate of the theoretical uncertainty.

In contrast to some previous approaches (e.g., Grossi et al. 2009; Desjacques et al. 2009), the good agreement with numerical simulations shown in Figs. 8 and 9 is obtained from Eq. (85) without any fitting parameter (such as the rescaling parameter $q$ in Grossi et al. 2009; or the mass function parameters in Desjacques et al. 2009). As for the halo mass function studied in Sect. 3.2, the role of this parameter is partly played by the use 

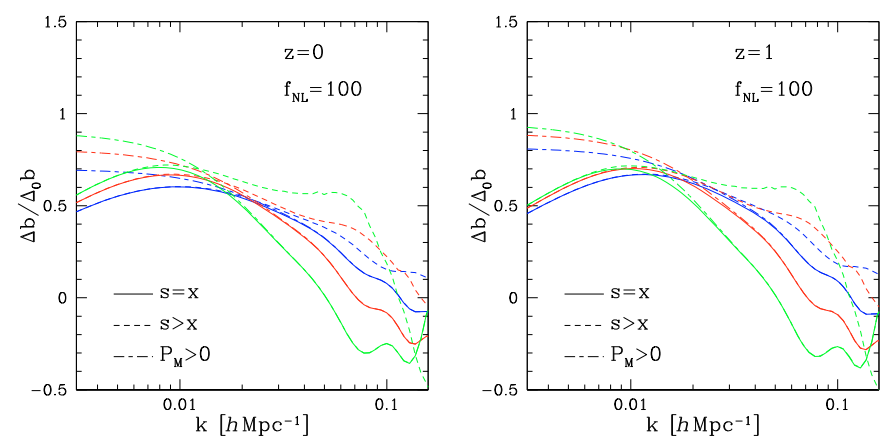

Fig. 10. The Fourier-space ratio $\Delta b_{M}\left(k, f_{\mathrm{NL}}\right) / \Delta_{0} b_{M}\left(k, f_{\mathrm{NL}}\right)$ of the correction $\Delta b_{M}\left(k, f_{\mathrm{NL}}\right)=b_{M}\left(k, f_{\mathrm{NL}}\right)-b_{M}(k, 0)$ to the Gaussian bias $b_{M}(k, 0)$, divided by the scaling factor $\Delta_{0} b_{M}$ defined in Eq. (94), as a function of wavenumber $k$. We show the case $f_{\mathrm{NL}}=100$ for several masses, $M=10^{13}$ (blue), $10^{14}$ (red), and $10^{15} h^{-1} M_{\odot}$ (green) at redshifts $z=0$ (left panel) and $z=1$ (right panel). The curves labeled " $s=x$ ", " $s>x$ ", and " $P_{M}>0$ ", correspond to Eqs. (85), (87), and (89), respectively.

of the exact linear threshold $\delta_{\mathrm{L}}=\mathcal{F}^{-1}(200)$, which is predicted by the spherical dynamics of the rare-event saddle points. This makes formulae such as Eq. (85) fully predictive for any values of cosmological parameters.

As in Fig. 6, in order to see the scaling of the correction $\Delta b_{M}\left(k, f_{\mathrm{NL}}\right)=b_{M}\left(k, f_{\mathrm{NL}}\right)-b_{M}(k, 0)$ to the Fourierspace Gaussian bias more clearly, we show the ratio $\Delta b_{M}\left(k, f_{\mathrm{NL}}\right) / \Delta_{0} b_{M}\left(k, f_{\mathrm{NL}}\right)$ in Fig. 10 , where we now define

$\Delta_{0} b_{M}\left(k, f_{\mathrm{NL}}\right)=f_{\mathrm{NL}} b_{M}(k, 0) \frac{2 \delta_{\mathrm{L}}}{\alpha(k)}$.

The correction obtained from a simple peak-background split argument instead gives $\Delta b_{M}\left(k, f_{\mathrm{NL}}\right)=f_{\mathrm{NL}}\left[b_{M}(k, 0)-1\right]\left(2 \delta_{\mathrm{L}} / \alpha(k)\right)$, as in Eq. (2), see Dalal et al. (2008); Slosar et al. (2008). However, in our formalism, both the leading term $\delta_{\mathrm{L}}^{2} / \sigma_{q}^{4}$ (third term in Eq. (85)) and the subleading term $\delta_{\mathrm{L}} / \sigma_{q}^{2}$ (first term in Eq. (85)) are modified by primordial non-Gaussianity (the terms in the brackets that follow). The term $\delta_{\mathrm{L}} / \sigma_{q}^{2}$ arises from the Lagrangian to Eulerian space mapping (the prefactor $\left(1+\delta_{\mathrm{L} M}\right)$ in Eq. (72)), and it corresponds to the factor 1 in the more usual Eq. (2), which is not modified in the simplest model. However, in general we can expect non-Gaussianities to also affect this Lagrangian to Eulerian space mapping, and our model gives an estimate of this effect through Eq. (40). Therefore, we scale $\Delta_{0} b_{M}\left(k, f_{\mathrm{NL}}\right)$ with $b_{M}(k, 0)$ rather than with $\left[b_{M}(k, 0)-1\right]$ in Eq. (94). We show the results obtained at redshifts $z=0$ and $z=1$ in Fig. 6 for several masses. The curves labeled " $s=x$ ", " $s>x$ " and " $P_{M}>0$ " correspond to Eqs. (85), (87), and (89) respectively. We can see that the collapse of the curves obtained for different masses is not exact, as could be expected since Eq. (85) is more complex than Eq. (94), but the scaling (94) still captures most of the dependence on the halo mass $M$. In fact, most of the dispersion seen in Fig. 6 does not arise from the different masses but from the various approximations (85), (87), and (89). This should provide an estimate of the theoretical uncertainty. As in Figs. 8 and 9, Eq. (89), which involves higher-order terms over $f_{\mathrm{NL}}$ to ensure that $P_{M}(k)$ is always positive, rises above Eq. (85) at low $k\left(k<0.01 h \mathrm{Mpc}^{-1}\right)$ and is indistinguishable at higher $k$, whereas Eq. (87) rises above (85) at high $k\left(k>0.01 h \mathrm{Mpc}^{-1}\right)$, where the correction $\Delta b_{M}\left(k, f_{\mathrm{NL}}\right)$ is quite small, and is indistinguishable at lower $k$. Approximations (89) and (87) are likely to be most accurate at low and high $k$, respectively, see also Figs. 8 and 9 . In any case, we can see that the scaling with wavenumber, $\Delta b_{M}\left(k, f_{\mathrm{NL}}\right) \sim 1 / \alpha(k)$, is slightly broken, although this still provides a good approximation. As in the numerical simulations of Desjacques et al. (2009), the ratio $\Delta b / \Delta_{0} b$ is suppressed at high $k$, which means that the correction $\Delta b_{M}\left(k, f_{\mathrm{NL}}\right)$ decreases slightly faster than $1 / \alpha(k)$ over the range $0.01-0.1 \mathrm{~h} \mathrm{Mpc}^{-1}$. In agreement with the real-space behavior seen in Fig. 6, it appears that the correction $\Delta b_{M}\left(k, f_{\mathrm{NL}}\right)$ scales slightly more closely as $k^{-2}$ than as $1 / \alpha(k)$ over this range, although neither of these two behaviors is exact.

\section{Conclusion}

We have shown in this article how to extend to non-Gaussian initial conditions the computation of the mass function and of the bias of dark matter halos presented in Valageas (2009b) for the Gaussian case. This relies on a saddle-point approach that allows to derive the high-mass asymptotic tails of the quantities of interest from the statistical weight of the initial conditions, supplemented by additional nonlinear constraints. Then, focusing on the case of " $f_{\mathrm{NL}}$-type" primordial non-Gaussianity, where the linear gravitational potential can be written as the sum of linear and quadratic terms over an auxiliary Gaussian field $\chi$, we explained how to obtain the relevant saddle-points as a perturbative series over the nonlinear parameter $f_{\mathrm{NL}}$. This method is very general, and it applies to any case of small primordial non-Gaussianity, where Bardeen's potential (or equivalently, below the Hubble radius, the gravitational potential or the density field) can be written as a polynomial over a Gaussian field $\chi$ as

$\Phi=\chi+\sum_{i=2}^{k} f_{i}\left(\chi^{i}-\left\langle\chi^{i}\right\rangle\right)$

where the nonlinear parameters $f_{i}$ are small. Then, following the method presented in Sects. 3, 4, the one-cell and two-cell saddle points (associated with the one-point and two-point density distributions, whence the halo mass function and bias) can be computed as a perturbative series over the coefficients $f_{i}$, which need not be of the same order. This includes the case of a cubic term $g_{\mathrm{NL}} \chi^{3}$ in particular. Of course, it also extends to the cases where the coefficients $f_{i}$ are not mere numbers but convolution kernels and to several Gaussian fields $\chi_{j}$ (which can have a nonzero cross-correlation). In all such cases, the highmass asymptotics are set by the statistical weight $\mathrm{e}^{-\left(\chi_{i} \cdot C_{i j}^{-1} \cdot \chi_{j}\right) / 2}$ of these Gaussian fields, taken at the saddle point associated with the maximization of this weight under appropriate nonlinear constraints that express the mapping from $\chi_{j}$ to the relevant quantity, such as the nonlinear density within a spherical cell.

Focusing on the case of local-type primordial nonGaussianity, we described how to obtain, up to linear order over $f_{\mathrm{NL}}$, the one-cell saddle point associated with the probability distributions $\mathcal{P}_{\mathrm{L}}\left(\delta_{\mathrm{L}}\right)$ and $\mathcal{P}(\delta)$ of the linear and nonlinear density contrasts within spherical cells. This gives the quasi-linear limit of these distributions, as well as the high-mass exponential falloff of the halo mass function. One advantage of our method is that it allows us to explicitly check that realistic amounts of primordial non-Gaussianity have no significant effect on the density profile of this saddle point. This ensures that shell crossing appears for (almost) the same nonlinear density $\delta_{+} \gtrsim 200$ (see Valageas 2009b), so that the high-mass tail of the halo mass function can be derived provided halos are defined by a nonlinear density threshold that is below this upper bound (which is indeed the case). Although this procedure only gives the highmass tail, we proposed a simple change of variable, applied to the mass function fitted to Gaussian numerical simulations, that 
obeys this high-mass asymptotic while keeping the mass function normalized to unity. If one uses the Press-Schechter mass function, this gives back the result of Matarrese et al. (2000), but we argue that this procedure is somewhat more natural if one wishes to recover a more accurate mass function in the Gaussian case. We also checked that this agrees with results from nonGaussian numerical simulations.

Next, we applied this method to the two-point correlation of massive halos, following the approach of Kaiser (1984). As in Valageas (2009b) we take the displacement of halo pairs under their mutual gravitational attraction into account. This gives the real-space halo correlation $\xi_{M}(x)$, whence the real-space bias $b_{M}(x)$. Since this approach does not assume that the halo correlation is weak, the nonlinear formula it yields can be used to check whether the "linearized" form (where one only keeps the linear term over the matter correlation $\xi(x))$ is a good approximation in the regime of interest. As expected, we find that the correction $\Delta b_{M}\left(x, f_{\mathrm{NL}}\right)$ to the Gaussian bias grows with $b_{M}(x, 0)$ and with scale, roughly as $f_{\mathrm{NL}} b_{M}(x, 0) x^{2}$, up to $x \sim 100 h^{-1} \mathrm{Mpc}$. Beyond this scale, the baryon acoustic oscillation and the fact that the halo and matter correlations do not change sign at the same point lead to strong oscillations and divergent spikes for $b_{M}(x)$. This means that, for $x>100 h^{-1} \mathrm{Mpc}$, the bias is no longer a useful quantity, and one should directly work with the halo and matter correlations. In agreement with Desjacques (2008), we find that the two-point correlation of massive halos, which have a large bias, strongly amplifies the baryon acoustic oscillation. In addition we also obtain the modifications associated with primordial non-Gaussianity. The baryon oscillation remains strongly amplified, with a small shift, but somewhat less so for positive $f_{\mathrm{NL}}$.

Finally, we used the "linearized" form of the halo two-point correlation to derive the halo power spectrum and the halo bias in Fourier space. We also give a simple recipe that ensures that the halo power spectrum always remains positive. (This only differs from the direct prediction by terms of order $f_{\mathrm{NL}}^{2}$ and higher.) We obtain good agreement with numerical simulations without introducing any free parameter. Moreover, the two formulae described above allow one to estimate the range over which linear approximations over $f_{\mathrm{NL}}$ are sufficient. Thus, we find that terms of order $f_{\mathrm{NL}}^{2}$ start playing a role at low $k\left(k<0.01 h \mathrm{Mpc}^{-1}\right)$ for large negative $f_{\mathrm{NL}}\left(f_{\mathrm{NL}}<-100\right)$, where the direct formula would give a negative power spectrum.

We also pointed out that the $k^{-2}$ behavior observed at low $k$ for the halo bias does not imply any divergence for the realspace two-point correlation. Indeed, this behavior is only obtained within a certain limit, and it is the halo power spectrum itself (i.e. $\Delta b^{2}$ rather than $\Delta b$ ) that shows this $k^{-2}$ factor. We showed that this is sufficient to explain the behavior observed in numerical simulations. Moreover, it avoids the need to introduce counterterms, that depend on the size of the survey, so as to obtain finite real-space correlations. This is an advantage of real-space approaches, such as the one presented in this paper, which are better suited to describing the nonlinear effects associated with the bias of massive halos.

These results, which do not involve free parameters except for the mass function (if one requires its full shape, where one needs the fit to numerical simulations for Gaussian initial conditions) should be useful for constraining primordial nonGaussianities from observations of large-scale structures. Thus, neither the high-mass tail of the halo mass function nor the bias require rescaling parameters (such as $\delta \rightarrow \delta_{\mathrm{c}} \sqrt{q}$ ), because such a correction to the linear threshold $\delta_{\mathrm{c}}$ is achieved through the use of the exact linear threshold $\delta_{\mathrm{L}}=\mathcal{F}^{-1}(200)$ predicted by the spherical dynamics of rare-event saddle points. This makes this approach more predictive than some of the previous works, since one does not need to run new simulations to fit for such $q$-factors in order to investigate other cosmologies. In particular, as discussed above for Eq. (95), the method presented in this article is quite general and can be applied to a large class of models. Moreover, since it provides results in both real space and Fourier space (i.e. the halo two-point correlation and power spectrum), it gives a complete and consistent description of halo clustering. As for previous approaches, the most reliable use of these models to constrain cosmology is to take advantage of the specific shape of the dependence on mass (for the mass function) or scale (for the bias) brought by primordial non-Gaussianity to constrain $f_{\mathrm{NL}}$, rather than the change in the amplitude at a given mass or scale.

\section{References}

Afshordi, N., \& Tolley, A. J. 2008, Phys. Rev. D, 78, 123507

Amara, A., \& Refregier, A. 2004, MNRAS, 351, 375

Arkani-Hamed, N., Creminelli, P., Mukohyama, S., \& Zaldarriaga, M. 2004, JCAP, 4, 1

Bardeen, J., Bond, J. R., Kaiser, N., \& Szalay, A. S. 1986, ApJ, 304, 15

Barnaby, N., \& Cline, J. M. 2006, Phys. Rev. D, 73, 106012

Bartolo, N., Matarrese, S., \& Riotto, A. 2002, Phys. Rev. D, 65, 103505

Bartolo, N., Komatsu, E., Matarrese, S., \& Riotto, A. 2004, Phys. Rep., 402, 103

Colafrancesco, S., Lucchin, F., \& Matarrese, S. 1989, ApJ, 345, 3

Dalal, N., Dore, O., Huterer, D., \& Shirokov, A. 2008, Phy. Rev. D, 77, 123514 Desjacques, V. 2008, Phys. Rev. D, 78, 103503

Desjacques, V., Seljak, U., \& Iliev, I. T. 2009, MNRAS, 396, 85

Falk, T., Rangarajan, R., \& Srednicki, M. 1993, ApJ, 403, L1

Grinstein, B., \& Wise, M. B. 1986, ApJ, 310, 19

Grossi, M., Dolag, K., Branchini, E., Matarrese, S., \& Moscardini, L. 2007, MNRAS, 382, 1261

Grossi, M., Verde, L., Carbone, C., et al. 2009, MNRAS, 398, 321

Hamilton, A. J. S., Kumar, P., Lu, E., \& Matthews, A. 1991, ApJ, 374, L1

Kaiser, N. 1984, ApJ, 284, L9

Komatsu, E., Dunkley, J., Nolta, M. R., et al. 2009, ApJS, 180, 330

Lucchin, F., \& Matarrese, S. 1988, ApJ, 330, L535

Lo Verde, M., Miller, A., Shandera, S., \& Verde, L. 2008, JCAP, 4, 14

Lyth, D. H., Ungarelli, C., \& Wands, D. 2000, Phys. Rev. D, 67, 023503

Maggiore, M., \& Riotto, A. 2009 [arXiv: 0903.1251]

Matarrese, S., \& Verde, L. 2008, ApJ, 677, L77

Matarrese, S., Lucchin, F., \& Bonometto, S. A. 1986, ApJ, 310, L21

Matarrese, S., Verde, L., \& Jimenez, R. 2000, ApJ, 541, L10

Mo, H. J., \& White, S. D. M. 1996, MNRAS, 282, 347

Mo, H. J., Jing, Y. P., \& White, S. D. M. 1997, MNRAS, 284, 189

Peacock, J. A., \& Dodds, S. J. 1996, MNRAS, 280, L19

Pillepich, A., Porciani, C., \& Hahn, O. 2010, MNRAS, 402, 191

Politzer, H. D., \& Wise, M. B. 1984, ApJ, 285, L1

Press, W. H., \& Schechter, P. 1974, ApJ, 187, 425

Senatore, L., Tassev, S., \& Zaldarriaga, M. 2009, JCAP, 9, 38

Slosar, A., Hirata, C., Seljak, U., Ho, S., \& Padmanabhan, N. 2008, JCAP, 8,31

Valageas, P. 2002a, A\&A, 382, 412

Valageas, P. 2002b, A\&A, 382, 431

Valageas, P. 2009a, Phys. Rev. E, 80, 016305

Valageas, P. 2009b, A\&A, 508, 93

Wands, D., \& Slosar, A. 2009, Phys. Rev. D, 79, 123507 